\title{
GLOBAL ENTROPY SOLUTIONS OF THE GENERAL NONLINEAR HYPERBOLIC BALANCE LAWS WITH TIME-EVOLUTION FLUX AND SOURCE*
}

\author{
SHIH-WEI CHOU ${ }^{\dagger}$, JOHN M. HONG ${ }^{\ddagger}$, AND YING-CHIN SU§
}

\begin{abstract}
In this paper we investigate the initial and initial-boundary value problems for strictly hyperbolic balance laws with time-evolution of flux and source. Such nonlinear balance laws arise in, for instance, gas dynamics equations in time-dependent ducts and nozzles, shallow water equations, lanes-changing model in traffic flow and Einstein's field equations in a spherically symmetric spacetime. To account for the time dependence of flux and source, we introduce the perturbed Riemann and boundary Riemann problems. Such Riemann problems have unique solutions within elementary waves and an additional family of waves. Based on the work of [12, 13], a new version of Glimm scheme is introduced and its stability is established by modified interaction estimates. Finally, the existence of global entropy solutions is achieved by showing the consistency of scheme, the weak convergence of source term and the entropy inequalities.
\end{abstract}

Key words. Initial value problem, initial-boundary value problem, hyperbolic conservation laws, nonlinear balance laws, entropy solutions, Riemann problem, perturbed Riemann problem, perturbed boundary Riemann problem, wave interaction estimates, generalized Glimm scheme.

AMS subject classifications. 35L60, 35L65, 35L67.

1. Introduction. In this paper we are concern with the initial and initialboundary value problems for strictly hyperbolic balance laws with time-evolution of flux and source:

$$
\begin{array}{rlrl}
(\mathrm{IVP}) \quad u_{t}+f(a(x, t), u)_{x} & =a_{x} g\left(a(x, t), a_{x}, a_{t}, u\right), & & (x, t) \in \mathbb{R} \times \mathbb{R}^{+}, \\
u(x, 0) & =u_{0}(x) \in \Omega, & & -\infty<x<\infty, \\
(\mathrm{IBVP}) \quad u_{t}+f(a(x, t), u)_{x} & =a_{x} g\left(a(x, t), a_{x}, a_{t}, u\right), & & (x, t) \in \mathbb{R}^{+} \times \mathbb{R}^{+}, \\
u(x, 0) & =u_{0}(x) \in \Omega, & x \geq 0, \\
u_{1}(0, t) & =u_{1 B}(t), & t>0,
\end{array}
$$

where $\Omega$ is a ball of radius $r$ in $\mathbb{R}^{2}, u=\left(u_{1}, u_{2}\right)$ is the unknown, $f=\left(f_{1}, f_{2}\right)$ and $g=\left(g_{1}, g_{2}\right)$ are smooth functions of their variables, and $u_{1 B}(t), u_{0}(x) \in L^{\infty} \cap B V$. In addition, $a(x, t) \in \mathbb{R}$ is assumed to be a given smooth function defined in $\mathbb{R} \times[0, \infty)$. The initial-boundary condition of (1.2a) is of Dirichlet type. Throughout this paper, we impose the following conditions.

$\left(\mathrm{A}_{1}\right)$ The total variations of $a(0, \cdot), a(\cdot, t)$ and $a_{t}(\cdot, t)$ are sufficiently small for every $t \geq 0$.

$\left(\mathrm{A}_{2}\right)$ (i) Each component of $R_{0}(a, u)$ is non-zero for all $(x, t)$ and $u \in \Omega$ where

$$
R_{0}(a, u):=\left(D_{u} f\right)^{-1}\left(g-f_{a}\right)(a, u),
$$

*Received August 15, 2011; accepted for publication July 17, 2012. Work partially supported by the National Science Council of Taiwan and Center for Theoretical Sciences, Mathematical Division, NCU.

${ }^{\dagger}$ Department of Mathematics, National Central University, Taoyuan 32001, Taiwan (962401002@ cc.ncu.edu.tw).

${ }^{\ddagger}$ Department of Mathematics, National Central University, Taoyuan 32001, Taiwan (jhong@math. ncu.edu.tw).

$\S$ Department of Mathematics, Fu Jen Catholic University, New Taipei 24205, Taiwan (ycsu@math. fju.edu.tw). 
(ii) $\frac{\partial f_{1}}{\partial u_{2}}$ is non-zero for all $a \in \mathbb{R}$ and $u \in \Omega$ in $\operatorname{IBVP}(1.2)$.

$\left(\mathrm{A}_{3}\right)$ Our system is strictly hyperbolic and the eigenvalues $\lambda_{1}(a, u)$ and $\lambda_{2}(a, u)$ of $D_{u} f$ satisfy

$$
\lambda_{1}(a, u)<0<\lambda_{2}(a, u) \quad \text { in } \mathbb{R} \times \Omega .
$$

This condition implies that the boundary in IBVP (1.2) is non-characteristic. We assume further that each characteristic field of (1.1a) is genuinely nonlinear, more precisely, for $i=1,2$,

$$
\nabla \lambda_{i}(a, u) \cdot R_{i}(a, u)=1 \quad \text { in } \mathbb{R} \times \Omega .
$$

Here $R_{i}$ is the normalized right eigenvector corresponding to $\lambda_{i}$.

One famous example for applications is the Euler equations of compressible isentropic gas dynamics

$$
\begin{aligned}
\partial_{t}(a \rho)+\partial_{x}(a \rho v) & =0, \\
\partial_{t}(a \rho v)+\partial_{x}\left(a \rho v^{2}+a p\right) & =a_{x} p,
\end{aligned}
$$

where $a=a(x, t)>0$ denote the spatial-time dependent cross section of a duct, and $\rho, v$ and $p=p(\rho)$ are the density, the velocity and the pressure of gas, respectively. Here we ignore the effect of gas flow through the duct, so that $a$ is given. The others can be found in the shallow water equations with time-variation of riverbed elevation [32], the traffic flow model with lanes-changing intensity [19] and the Einstein's field equations for a spherically symmetric space-time [11]

$$
\begin{aligned}
u_{t}+f(u, A, B)_{x} & =\bar{g}\left(u, A, B, A_{x}, B_{x}, B_{t}, x\right), \\
A_{x} & =h^{0}(u, A, B, x), \\
B_{x} & =h^{1}(u, A, B, x),
\end{aligned}
$$

where $u$ is related to the stress energy tensor $T$, and $A$ and $B$ are the elements of metric in the standard Schwarzschild coordinates. To treat (1.1a) as a warm up model of (1.5), we need to solve the second and the third equations of (1.5) for $A$ and $B$ in advance, then writing $\bar{g}$ as $A_{x} g$ (or $B_{x} g$ ) when $A_{x}$ (or $B_{x}$ ) is non-zero.

In this paper, we will establish the existence of global entropy solutions by using a Glimm-type method. Our results can be extended to the general $n \times n$ systems in the same analysis. Therefore, we only consider the $2 \times 2$ case here.

We review some results on the subject and clarify the motivation of the study. The Riemann problem for $n \times n$ strictly hyperbolic conservation laws

$$
u_{t}+f(u)_{x}=0 \text {, }
$$

was first studied by Lax [21]. The solution is obtained by resolving the jump discontinuity of initial data into fans of elementary waves (including rarefaction waves, shocks and contact discontinuities), that is, the solution is self-similar and consists of at most $n+1$ constant states separated by these waves. The existence of BV solutions to a general Cauchy problem with initial data of small total variation is established by Glimm [9]. The solution is attained as the $\Delta x \downarrow 0$ limit of a family of approximate solutions $u_{\Delta x}(x, t)$ which are constructed in time steps of length $\Delta t=O(\Delta x)$ by the following procedure: Assuming that $u_{\Delta x}(x, t)$ has already determined on $\mathbb{R} \times[0, n \Delta t)$, 
one constructs initial data $u_{\Delta x}(x, n \Delta t)$ as a random step function approximation to $u_{\Delta x}\left(x, n \Delta t^{-}\right)$and then obtains $u_{\Delta x}(x, t)$ in the next time strip $\mathbb{R} \times[n \Delta t,(n+1) \Delta t)$ by Lax's method. A random choice of initial data in each time step ensures the scheme is consistent, that is, the limit of $\left\{u_{\Delta x}(x, t)\right\}$ is a weak solution of (1.6). The convergence of the family $\left\{u_{\Delta x}(x, t)\right\}$, or a subfamily thereof, is secured by an a priori bound, independent of $\Delta x$, on the total variation, which is induced by approximate conservation laws that govern elementary wave interactions and is established with the help of Glimm functional. This scheme is established in the genuinely nonlinear case and generalized to the linearly degenerate case in the paper [27] referenced by Liu. The initial-boundary value problem for (1.6) with the shape of different boundaries was first studied by Goodman [10]. The author proved the global existence of weak solutions when initial and boundary data satisfy the so-called smallness and non-degeneracy conditions.

For the Cauchy problem of quasi-linear hyperbolic system

$$
u_{t}+f(x, u)_{x}=g(x, u),
$$

the global existence and asymptotic behavior of solutions were first established by Liu [26] by a steady state scheme. For further results on the initial-boundary value problems for (1.6) and (1.7), we refer to [1, 2, 3, 7, 8, 25, 28].

For the general strictly hyperbolic system

$$
u_{t}+f(u, x, t)_{x}=g(u, x, t),
$$

the Cauchy problem was studied by Dafermos-Hsiao [5] and Hong-LeFloch [13]. In [5], the authors use Glimm's scheme along with the method of fractional steps to construct a BV weak solution. An additional dissipativity condition on the flux and the source is imposed, that is, assume that there exists a constant $b>0$ such that

$$
R^{\mu \mu}(x, t)-\sum_{\kappa \neq \mu}\left|R^{\kappa \mu}(x, t)\right| \geq b, \quad \mu=1, \cdots, n,
$$

for every $(x, t) \in(-\infty, \infty) \times[0, \infty)$, where $R(x, t)=\left(R^{\kappa \mu}(x, t)\right):=-\left(r^{-1} g_{u} r\right)(0, x, t)$ and $r$ is the $n \times n$ matrix consisting of normalized right eigenvectors of $D_{u} f$. On the other hand, an alternative version of Glimm scheme for (1.8) was introduced in [13] whose Riemann solutions were constructed by the techniques of asymptotic expansion to the classical Riemann solutions and the frozen variables $(x, t)$ in $f$. Through the detailed wave interaction estimates, the global existence of entropy solutions for (1.8) was established provided that the $L^{1}$ norms of $\frac{\partial^{2} A}{\partial t \partial u}, \frac{\partial^{2} A}{\partial x \partial u}, q$ and $\frac{\partial q}{\partial u}$ are sufficiently small where $A:=D_{u} f$ and $q(t, x, u):=g(t, x, u)-\frac{\partial f}{\partial x}(t, x, u)$.

For the systems of nonlinear balance laws in the degenerate form

$$
\begin{aligned}
a_{t} & =0, \\
u_{t}+f(a(x), u)_{x} & =a^{\prime} g(a(x), u),
\end{aligned}
$$

the general $n \times n$ strictly hyperbolic case was first studied by LeFloch [23]. In [23], the addition of the extra equation $a_{t}=0$ allows us to consider (1.10) as a nonconservative system so that the results in [5] can be applied. On the other hand, the generalized Glimm method for (1.10) was given in [12] that the residual only converges weakly in $L^{1}$. The Riemann solutions in [12] are "weaker than weak" because, due to the re-scaling of the source by discontinuities, the Riemann solutions do not solve 
the equations even weakly, yet the Glimm scheme is a valid method and converges. The $2 \times 2$ resonant systems in the form of (1.10) was first studied by Isaacson and Temple [18]. The method in [18] showed that incorporating the source term as a wave gave sharp time independent bounds for solutions of the initial value problem, while the operator-splitting method gave only time dependent bounds in this nonstrictly hyperbolic setting. Recently, this framework was extended to quasi-linear wave equations $[4,14,29]$,

$$
u_{t t}-\left(p\left(\rho(x), u_{x}\right)\right)_{x}=\rho(x) h\left(\rho(x), u, u_{x}\right)
$$

with applications to shallow water wave and the deformation of rubbery materials. In [29], $\rho$ is considered as a more general form $\rho=\rho(x, t)$, and the result of global existence was obtained under a dissipative condition. The initial-boundary value problem for (1.10) was also studied in [15]. Some problems with regard to non-strictly hyperbolic and non-conservative systems, we refer to $[6,16,17,20,22,24,30]$.

In this paper, we investigate the possibility that time dependent sources can be treated like source free equations by incorporating an additional family of waves, and to utilize this in the Glimm scheme. Since the time dependence of $a$ is allowed, the framework in $[12,18]$ can only be carried out locally. To apply the method, the Riemann problems must account not only for the $x$-dependence of the source, but also for the time dependence. This then requires an approximate Riemann solver to account for time dependence. Since no total variation bound on the source in time is assumed, Glimm's functional in [9] may fail to be non-increasing in time. This will lead to the instability of Glimm method. Moreover, for approximate solutions $\left\{a_{\Delta x}^{\varepsilon}, u_{\Delta x}^{\varepsilon}\right\}$ generated by Glimm's scheme, it cannot be expected that $\left(\partial_{x} a_{\Delta x}^{\varepsilon}\right) \cdot g\left(a_{\Delta x}^{\varepsilon}, \partial_{x} a_{\Delta x}^{\varepsilon}, \partial_{t} a_{\Delta x}^{\varepsilon}, u_{\Delta x}^{\varepsilon}\right)$ does converge to $a_{x} g\left(a, a_{x}, a_{t}, u\right)$ in the weak sense.

In order to overcome difficulties above, the steps in the paper are thus as follows: (1) "weaker than weak" solutions of the Riemann and boundary Riemann problems are established that account for the leading order effects of time dependence in the source; (2) a modified Glimm-type interaction estimate and a boundary interaction estimate are obtained; (3) a Glimm-type argument is developed to prove time independent total variation estimates for the approximate solutions. At this stage, an additional assumption on $a(x, t)$ is necessary to obtain the total variation bounds of approximate solutions. (4) Finally, the weak convergence of the residual and

$$
\iint_{t>0}\left\{\partial_{x} a_{\Delta x}^{\varepsilon} \cdot g\left(a_{\Delta x}^{\varepsilon}, \bar{a}_{x}, b^{\varepsilon}, u_{\Delta x}^{\varepsilon}\right)-a_{x} g\left(a, a_{x}, a_{t}, u\right)\right\} \phi d x d t \rightarrow 0 \quad \text { as } \varepsilon, \Delta x \rightarrow 0
$$

for $\phi \in C_{c}^{1}$ are established to prove the Glimm method converges to a weak solution (modulo the usual subsequences). Here, $\bar{a}_{x}$ and $b^{\varepsilon}$ are given in Section 5. Here we point out that condition $\left(A_{2}\right)$ gives the existence and generic structure of the standing wave discontinuities for the Riemann and boundary Riemann problems [12]. Comparing to the results in $[5,12,29]$, the contribution of this paper is that the global existence result can be extended to more general flux and source without the dissipative condition.

We now give the definitions of weak and entropy solutions to the problems (1.1) and (1.2), and state the main theorem.

Definition 1.1. Let $E:=[0, \infty) \times[0, \infty)$ and $E_{*}:=(-\infty, \infty) \times[0, \infty) . W e$ say that a bounded measurable function $u$ is a weak solution to (1.2) if $u$ satisfies 
$R_{\phi}(a, u)=0$ for all $\phi \in C_{c}^{1}(E)$ where

$$
\begin{aligned}
R_{\phi}(a, u):= & \iint_{E} u \phi_{t}+f(a, u) \phi_{x}+a_{x} g\left(a, a_{x}, a_{t}, u\right) \phi d x d t+\int_{0}^{\infty} u_{0}(x) \phi(x, 0) d x \\
& +\int_{0}^{\infty} f(a, u)(0, t) \phi(0, t) d t .
\end{aligned}
$$

Similarly, $u$ is a weak solution to (1.1) if for all $\phi \in C_{c}^{1}\left(E_{*}\right)$,

$$
\iint_{E_{*}} u \phi_{t}+f(a, u) \phi_{x}+a_{x} g\left(a, a_{x}, a_{t}, u\right) \phi d x d t+\int_{-\infty}^{\infty} u_{0}(x) \phi(x, 0) d x=0 .
$$

Definition 1.2. Let $\mathcal{U}$ be a convex subset of $\mathbb{R}^{2}$, and let $U: \mathcal{U} \rightarrow \mathbb{R}$ and $F: \mathbb{R} \times \mathcal{U} \rightarrow \mathbb{R}$. We say that $(U(u), F(a, u))$ is an entropy pair of (1.1a) if $U$ is convex on $\mathcal{U}$, and $U, F$ satisfy

$$
D_{u} F=\left(D_{u} U\right)\left(D_{u} f\right) \text { on } \mathbb{R} \times \mathcal{U} .
$$

Furthermore, $u$ is called an entropy solution if $u$ is the weak solution of (1.2) and satisfies

$$
\begin{aligned}
& \iint_{E}\left[U(u) \phi_{t}+F(a, u) \phi_{x}+a_{x}\left(D_{u} U\left(g-f_{a}\right)+F_{a}\right) \phi\right] d x d t \\
& +\int_{0}^{\infty} U\left(u_{0}(x)\right) \phi(x, 0) d x+\int_{0}^{\infty} F(a, u)(0, t) \phi(0, t) d t \geq 0
\end{aligned}
$$

for every entropy pair $(U, F)$ and positive $\phi \in C_{c}^{1}(E)$. The definition for the entropy solutions of (1.1) can be given analogously.

MAIN THEOREM. Consider initial value problem (1.1) and initial-boundary value problem (1.2), where $a, f$ and $g$ satisfy conditions $\left(A_{1}\right)-\left(A_{3}\right)$. For a domain $\Sigma$, we define

$$
\omega(\Sigma):=\left\|a_{t}\right\|_{L^{1}(\Sigma)}+\left\|a_{x t}\right\|_{L^{1}(\Sigma)}
$$

Assume that $\omega(E), \omega\left(E_{*}\right)$ and the total variations of $u_{0}$ and $u_{1 B}$ are sufficiently small, where $E, E_{*}$ are in Definition 1.1. Let $\left\{\bar{u}_{\theta, \Delta x}^{\varepsilon}\right\}$ and $\left\{u_{\theta, \Delta x}^{\varepsilon}\right\}$ be respectively the sequence of approximate solutions for (1.1) and (1.2) by the generalized Glimm scheme. Then there exists a null set $N \subset \Phi$ and a sequence $\left\{\Delta x_{i}\right\} \rightarrow 0$ such that if $\theta \in \Phi \backslash N$, then $\bar{u}(x, t), u(x, t)$ are respectively the entropy solutions to (1.1) and (1.2), where

$$
\bar{u}(x, t):=\lim _{\Delta x_{i}, \varepsilon \rightarrow 0} \bar{u}_{\theta, \Delta x_{i}}^{\varepsilon}, \quad u(x, t):=\lim _{\Delta x_{i}, \varepsilon \rightarrow 0} u_{\theta, \Delta x_{i}}^{\varepsilon}
$$

This paper is organized as follows. In Section 2, to account for time dependence, we introduce a perturbed Riemann problem by asymptotic expansions of the flux and the source. Its approximate solution is constructed within elementary waves and an additional family of waves by the modified Lax method. In Section 3, we extend the results of Section 2 and [15] to the boundary Riemann problem. At the same time, the residual of approximate solutions in each grid is estimated for the consistency of our scheme. In Section 4, a generalized Glimm scheme (GGS for short) with boundary condition will be described. Moreover, the generalized versions of interaction estimates are also obtained. In Section 5, the main theorem is proved by the consistency of the GGS, the weak convergence of the source term and the entropy inequalities for the solution. 
2. Generalized Riemann solver and its error estimate. In this section we give a generalized Riemann solver for (1.1a). To start, for given $\left(x_{0}, t_{0}\right) \in \mathbb{R}^{+} \times[0, \infty)$ and $\delta, \kappa>0$ sufficiently small, we consider the following Riemann problem:

$$
\left\{\begin{array}{l}
u_{t}+f(a, u)_{x}=a_{x} g\left(a, a_{x}, a_{t}, u\right), \quad(x, t) \in\left(x_{0}-\kappa, x_{0}+\kappa\right) \times\left(t_{0}, t_{0}+\delta\right), \\
u\left(x, t_{0}\right)= \begin{cases}u_{L}, & x_{0}-\kappa \leq x<x_{0} \\
u_{R}, & x_{0}<x \leq x_{0}+\kappa,\end{cases}
\end{array}\right.
$$

where $a, f$ and $g$ satisfy conditions $\left(A_{1}\right)-\left(A_{3}\right)$, and $u_{L}$ and $u_{R} \in \Omega$ are two nearby constant states. When $a=a(x)$ and $g=g(a, u)$, by the results of [12], (2.1) can be treated like the source free equations by incorporating a linear degenerate wave field. Based on this observation, we utilize linear expansions of $a, a_{x}$ and $a_{t}$ in time $t=t_{0}$ to achieve a perturbed Riemann problem (PRP), which is derived as follows.

First, we approximate $a, a_{x}$ and $a_{t}$ in $\left(x_{0}-\kappa, x_{0}+\kappa\right) \times\left(t_{0}, t_{0}+\delta\right)$ by

$$
a(x, t) \approx \bar{a}(x)+\delta b(x), \quad a_{x}(x, t) \approx \bar{a}^{\prime}(x)+\delta b^{\prime}(x), \quad a_{t}(x, t) \approx b(x)+\delta c(x),
$$

respectively, where

$$
\bar{a}(x):=a\left(x, t_{0}\right), \quad b(x):=a_{t}\left(x, t_{0}\right), \quad c(x):=a_{t t}\left(x, t_{0}\right) .
$$

Then, $f$ and $g$ are approximated by

$$
\begin{aligned}
f(a, u) & \approx f(\bar{a}, u)+\delta b(x) f_{a}(\bar{a}, u) \\
g\left(a, a_{x}, a_{t}, u\right) & \approx\left(g+\delta b(x) g_{a}+\delta b^{\prime}(x) g_{a_{x}}+\delta c(x) g_{a_{t}}\right)\left(\bar{a}(x), \bar{a}^{\prime}(x), b(x), u\right),
\end{aligned}
$$

respectively, where $g_{a_{x}}:=\frac{\partial g}{\partial a_{x}}$ and $g_{a_{t}}:=\frac{\partial g}{\partial a_{t}}$. By (2.2), (2.4), (2.5) and omitting $O\left(\delta^{2}\right)$ terms, the system in $(2.1)$ is linearized to

(2.6) $u_{t}+\left[\left(f+\delta b(x) f_{a}\right)(\bar{a}, u)\right]_{x}=\bar{a}^{\prime}\left[g+\delta\left(b(x) g_{a}+b^{\prime}(x) g_{a_{x}}+c(x) g_{a_{t}}\right)\right]+\delta b^{\prime}(x) g$,

where $g, g_{a}, g_{a_{x}}$ and $g_{a_{t}}$ are all evaluated at $\left(\bar{a}(x), \bar{a}^{\prime}(x), b(x), u\right)$. Next, for $0<\varepsilon \ll 1$, the initial data $u\left(x, t_{0}\right)$ together with $\bar{a}(x)$ and $b(x)$ are approximated by

$$
\begin{gathered}
u^{\varepsilon}\left(x, t_{0}\right):= \begin{cases}u_{L}, & x_{0}-\kappa \leq x<x_{0}-\varepsilon \kappa, \\
\psi^{\varepsilon}(x), & \left|x-x_{0}\right| \leq \varepsilon \kappa, \\
u_{R}, & x_{0}+\varepsilon \kappa<x \leq x_{0}+\kappa,\end{cases} \\
\left(\bar{a}^{\varepsilon}(x), b^{\varepsilon}(x)\right):= \begin{cases}\left(a_{L}, b_{L}\right), & x_{0}-\kappa \leq x<x_{0}-\varepsilon \kappa, \\
\left(\eta^{\varepsilon}(x), a_{t}\left(x, t_{0}\right)\right), & \left|x-x_{0}\right| \leq \varepsilon \kappa, \\
\left(a_{R}, b_{R}\right), & x_{0}+\varepsilon \kappa<x \leq x_{0}+\kappa,\end{cases}
\end{gathered}
$$

where $\left(a_{L}, b_{L}\right)$ and $\left(a_{R}, b_{R}\right)$ are constant states, $\psi^{\varepsilon}(x)$ is a monotone function connecting $u_{L}$ at $x=x_{0}-\varepsilon \kappa$ and $u_{R}$ at $x=x_{0}+\varepsilon \kappa$, respectively, and $\eta^{\varepsilon}(x)$ is also a monotone function connecting $a_{L}$ at $x=x_{0}-\varepsilon \kappa$ and $a_{R}$ at $x=x_{0}+\varepsilon \kappa$, respectively. We then obtain the following perturbed Riemann problem centered at $\left(x_{0}, t_{0}\right)$ for $0<\varepsilon \ll 1$ :

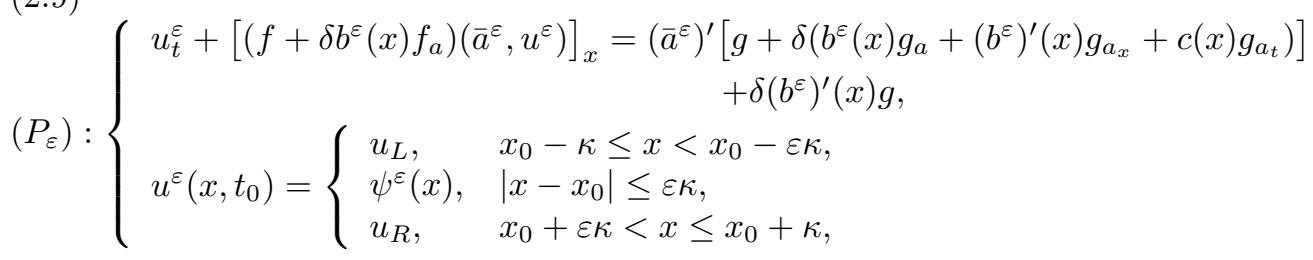


where $g, g_{a}, g_{a_{x}}$ and $g_{a_{t}}$ are all evaluated at $\left(\bar{a}^{\varepsilon}(x), \bar{a}^{\prime}(x), b^{\varepsilon}(x), u^{\varepsilon}\right)$, and $\bar{a}^{\varepsilon}(x)$ and $b^{\varepsilon}(x)$ are given in (2.8). The limiting perturbed Riemann problem, denoted by $\left(P_{0}\right)$, is given by taking the limit $\varepsilon \rightarrow 0$ to $\left(P_{\varepsilon}\right)$. The system in $\left(P_{\varepsilon}\right)$ is still genuinely nonlinear for small $\delta>0$. To see this, we define

$$
f^{\delta}(a, b, u):=f(a, u)+\delta b f_{a}(a, u) .
$$

Let $\left\{\lambda_{i}^{\delta}(a, b, u)\right\}_{i=1}^{2}$ be the eigenvalues of the Jacobian matrix $D_{u} f^{\delta}$ and let $\left\{R_{i}^{\delta}(a, b, u)\right\}_{i=1}^{2}$ be the corresponding right eigenvectors. Then there exist bounded functions $\left\{k_{i}(a, b, u)\right\}_{i=1}^{2}$ and $\left\{K_{i}(a, b, u)\right\}_{i=1}^{2}$ such that

$$
\lambda_{i}^{\delta}=\lambda_{i}(a, u)+\delta k_{i}, \quad R_{i}^{\delta}=R_{i}(a, u)+\delta K_{i}, \quad i=1,2 .
$$

By (2.11) and condition $\left(A_{3}\right)$, we have for sufficiently small $\delta$

$$
\begin{aligned}
\left(\nabla \lambda_{i}^{\delta} \cdot R_{i}^{\delta}\right)(a, b, u) & =\left(\nabla \lambda_{i} \cdot R_{i}\right)(a, u)+\delta\left(\nabla \lambda_{i} \cdot K_{i}+\nabla k_{i} \cdot\left(R_{i}+\delta K_{i}\right)\right)(a, b, u) \\
& =\left(\nabla \lambda_{i} \cdot R_{i}\right)(a, u)+O(\delta)>0, \quad i=1,2,
\end{aligned}
$$

which implies that the characteristic fields of (2.9) are genuinely nonlinear.

Now, we construct approximate solutions to (2.9) by modified Lax's method. Let

$$
\begin{aligned}
& D_{L}^{\varepsilon}:=\left[x_{0}-\kappa, x_{0}-\varepsilon \kappa\right) \times\left[t_{0}, t_{0}+\delta\right], \quad D_{R}^{\varepsilon}:=\left(x_{0}+\varepsilon \kappa, x_{0}+\kappa\right] \times\left[t_{0}, t_{0}+\delta\right], \\
& D_{M}^{\varepsilon}:=\left[x_{0}-\varepsilon \kappa, x_{0}+\varepsilon \kappa\right] \times\left(t_{0}, t_{0}+\delta\right], \quad \Gamma_{\varepsilon}:=\left[x_{0}-\varepsilon \kappa, x_{0}+\varepsilon \kappa\right] \times\left\{t_{0}\right\} .
\end{aligned}
$$

Since $\bar{a}^{\varepsilon}$ and $b^{\varepsilon}$ are constants in $D_{L}^{\varepsilon}$ and $D_{R}^{\varepsilon},(2.9)$ is reduced to

$$
\left\{\begin{array}{l}
u_{t}^{\varepsilon}+f^{\delta}\left(a_{L}, b_{L}, u^{\varepsilon}\right)_{x}=0, \quad \text { on }\left(D_{L}^{\varepsilon}\right)^{o}, \\
u_{t}^{\varepsilon}+f^{\delta}\left(a_{R}, b_{R}, u^{\varepsilon}\right)_{x}=0, \quad \text { on }\left(D_{R}^{\varepsilon}\right)^{o}, \\
u^{\varepsilon}\left(x, t_{0}\right)= \begin{cases}u_{L}, & x_{0}-\kappa \leq x<x_{0}-\varepsilon \kappa, \\
u_{R}, & x_{0}+\varepsilon \kappa<x \leq x_{0}+\kappa,\end{cases}
\end{array}\right.
$$

where $\left(D_{L}^{\varepsilon}\right)^{o}$ and $\left(D_{R}^{\varepsilon}\right)^{o}$ denote the interiors of $D_{L}^{\varepsilon}$ and $D_{R}^{\varepsilon}$, respectively. Owing to the genuinely nonlinearity of (2.14), either a rarefaction wave or a shock wave is adopted as the solution in every characteristic field. Let $\psi_{j}^{\delta}, j=1,2$, denote the jth wave curve combined with either a rarefaction wave or a shock of the jth family. Then, by (2.11) and the results of [21], $\psi_{1}^{\delta}$ is parametrized as

$$
\begin{aligned}
\psi_{1}^{\delta}\left(\eta_{1} ; a_{L}, b_{L}, u_{L}\right) \\
=u_{L}+\eta_{1} R_{1}^{\delta}\left(a_{L}, b_{L}, u_{L}\right)+\frac{\eta_{1}^{2}}{2}\left(R_{1}^{\delta} \cdot \nabla R_{1}^{\delta}\right)\left(a_{L}, b_{L}, u_{L}\right)+O\left(\left|\eta_{1}\right|^{3}\right) \\
=u_{L}+\eta_{1} R_{1}\left(a_{L}, u_{L}\right)+\frac{\eta_{1}^{2}}{2}\left(R_{1} \cdot \nabla R_{1}\right)\left(a_{L}, u_{L}\right) \\
\quad+\left(\eta_{1} \delta K_{1}+\frac{\eta_{1}^{2}}{2} \delta K_{1} \cdot \nabla R_{1}+\frac{\eta_{1}^{2}}{2} \delta R_{1} \cdot \nabla K_{1}+\frac{\eta_{1}^{2}}{2} \delta^{2} K_{1} \cdot \nabla K_{1}\right)\left(a_{L}, b_{L}, u_{L}\right) \\
\quad+O\left(\left|\eta_{1}\right|^{3}\right),
\end{aligned}
$$

where $\eta_{1}$ is the (signed) wave strength of $\psi_{1}^{\delta}$. If $\psi_{1}^{\delta}$ is the rarefaction wave, then $\eta_{1}$ is a function of $\frac{x-x_{0}+\varepsilon \kappa}{t-t_{0}}$ and $\eta_{1}>0$. If $\psi_{1}^{\delta}$ is the shock, then $\eta_{1}<0$. In addition, the Rankine-Hugoniot condition of (2.14) gives

$$
s_{1}^{\delta}\left[\psi_{1}^{\delta}\right]=\left[f^{\delta}\left(a_{L}, b_{L}, \psi_{1}^{\delta}\right)\right]=\left[f\left(a_{L}, \psi_{1}^{\delta}\right)\right]+\left[\delta b_{L} f_{a}\left(a_{L}, \psi_{1}^{\delta}\right)\right],
$$


where $s_{1}^{\delta}$ is the speed of the shock-front and [.] denotes the difference of states across the shock. Notice that $\psi_{1}^{\delta}$ is independent of $\varepsilon$ and the limit

$$
\begin{aligned}
\psi_{1}\left(\eta_{1} ; a_{L}, u_{L}\right) & :=\lim _{\varepsilon, \delta \rightarrow 0} \psi_{1}^{\delta}\left(\eta_{1} ; a_{L}, b_{L}, u_{L}\right) \\
& =u_{L}+\eta_{1} R_{1}\left(a_{L}, u_{L}\right)+\frac{\eta_{1}^{2}}{2}\left(R_{1} \cdot \nabla R_{1}\right)\left(a_{L}, u_{L}\right)+O\left(\left|\eta_{1}\right|^{3}\right)
\end{aligned}
$$

is the 1 -wave solution of $u_{t}+f\left(a_{L}, u\right)_{x}=0$. Similarly, the curves of 2-rarefaction wave and 2-shock in $D_{R}^{\varepsilon}$ can be proved as in (2.15) except that $a_{L}$ and $b_{L}$ are replaced by $a_{R}$ and $b_{R}$.

It remains to construct approximate solutions of $(2.9)$ in $D_{M}^{\varepsilon}$. Since $\bar{a}^{\varepsilon}=\eta^{\varepsilon}(x)$ is only $x$-dependent, we augment $(2.9)$ by adding $\bar{a}_{t}^{\varepsilon}=0$. By the framework of [12], an additional family of waves can be treated as waves of the zero characteristic field. To achieve the target, we can further approximate $b^{\varepsilon}(x)$ as

$$
b^{\varepsilon}(x) \approx b^{\varepsilon}\left(x_{0}\right)+\left(x-x_{0}\right)\left(b^{\varepsilon}\right)^{\prime}\left(x_{0}\right)
$$

and apply this to (2.9). Let $u_{t}^{\varepsilon}$ vanish in (2.9) and omit $O(1) \delta\left|x-x_{0}\right|$ terms, then we obtain a time-independent approximate solution (standing wave) $u_{s}^{\varepsilon}(x)$ in $D_{M}^{\varepsilon}$ which satisfies the following ordinary differential equations:

$$
\begin{aligned}
{\left[\left(f+\delta b^{\varepsilon}\left(x_{0}\right) f_{a}\right)\left(\eta^{\varepsilon}, u_{s}^{\varepsilon}\right)\right]_{x}=} & \left(\eta^{\varepsilon}\right)^{\prime}\left\{g+\delta\left(b^{\varepsilon}\left(x_{0}\right) g_{a}+\left(b^{\varepsilon}\right)^{\prime}\left(x_{0}\right) g_{a_{x}}+c\left(x_{0}\right) g_{a_{t}}\right)\right\} \\
& +\delta\left(b^{\varepsilon}\right)^{\prime}\left(x_{0}\right)\left(g-f_{a}\right),
\end{aligned}
$$

where $g, g_{a}, g_{a_{x}}$ and $g_{a_{t}}$ are evaluated at $\left(\eta^{\varepsilon}(x), \bar{a}^{\prime}(x), b^{\varepsilon}(x), u_{s}^{\varepsilon}\right)$, and $\bar{a}(x)$ is given in (2.3). Since $\eta^{\varepsilon}(x)$ is monotone, by the inverse function theorem, $x$ can be expressed as a function of $\eta^{\varepsilon}$ and there exists a nonzero continuous function $q$ such that $q\left(\eta^{\varepsilon}\right)=$ $\left(\frac{d \eta^{\varepsilon}}{d x}\right)^{-1}$. Applying the re-scaling to (2.19), we would have the following initial value problem:

$$
\left\{\begin{aligned}
\frac{d u_{s}^{\varepsilon}}{d \eta^{\varepsilon}} & =\widetilde{R}_{0}\left(\eta^{\varepsilon}, u_{s}^{\varepsilon}, x_{0}, t_{0} ; \eta_{1}\right)+\delta \widetilde{L}\left(\eta^{\varepsilon}, u_{s}^{\varepsilon}, x_{0}, t_{0} ; \eta_{1}\right), \\
u_{s}^{\varepsilon}\left(a_{L}\right) & =u_{1},
\end{aligned}\right.
$$

where

$$
\begin{aligned}
\widetilde{R}_{0} & :=\left(D_{u} f+\delta b^{\varepsilon}\left(x_{0}\right) D_{u} f_{a}\right)^{-1} \cdot\left(N_{1}+N_{2}\right), \\
\widetilde{L} & :=\left(D_{u} f+\delta b^{\varepsilon}\left(x_{0}\right) D_{u} f_{a}\right)^{-1} \cdot\left(b^{\varepsilon}\right)^{\prime}\left(x_{0}\right) q\left(\eta^{\varepsilon}\right)\left(g-f_{a}\right), \\
N_{1} & :=g-f_{a}+\delta b^{\varepsilon}\left(x_{0}\right)\left(g_{a}-f_{a a}\right), \quad N_{2}:=\delta\left(\left(b^{\varepsilon}\right)^{\prime}\left(x_{0}\right) g_{a_{x}}+c\left(x_{0}\right) g_{a_{t}}\right),
\end{aligned}
$$

and the initial data $u_{1}$ satisfies

$$
u_{1}=u_{L}+\eta_{1} R_{1}^{\delta}\left(a_{L}, b_{L}, u_{L}\right)+O\left(\left|\eta_{1}\right|^{2}\right) .
$$

It is noticed that $D_{u} f+\delta b^{\varepsilon}\left(x_{0}\right) D_{u} f_{a}$ is non-singular for small $\delta$, and that $\delta \widetilde{L}$ in $(2.20)$ is of order $\varepsilon \delta \kappa$ by the facts that $q\left(\eta^{\varepsilon}\right)=\left(\frac{d \eta^{\varepsilon}}{d x}\right)^{-1}=O(\varepsilon \kappa)$ and $\widetilde{L}$ is bounded.

LEMmA 2.1. For sufficiently small $\delta>0$, there exist vectors $J_{1}, J_{2}$ and a matrix $J_{*}$ evaluated at $\left(\eta^{\varepsilon}, u_{s}^{\varepsilon}, x_{0}, t_{0}\right)$ such that

$$
\widetilde{R}_{0}\left(\eta^{\varepsilon}, u_{s}^{\varepsilon}, x_{0}, t_{0} ; \eta_{1}\right)=R_{0}\left(\eta^{\varepsilon}, u_{s}^{\varepsilon}\right)+\delta\left(J_{1}+J_{2}\right),
$$


where $R_{0}$ is given in $\left(A_{2}\right)$,

$$
\begin{aligned}
& J_{1}:=-b^{\varepsilon}\left(x_{0}\right)\left(D_{u} f\right)^{-1} J_{*}\left(N_{1}+N_{2}\right), \\
& J_{2}:=\left(D_{u} f\right)^{-1}\left\{b^{\varepsilon}\left(x_{0}\right)\left(g_{a}-f_{a a}\right)+\left(b^{\varepsilon}\right)^{\prime}\left(x_{0}\right) g_{a_{x}}+c\left(x_{0}\right) g_{a_{t}}\right\}, \\
& J_{*}:=\sum_{i=0}^{\infty}(-1)^{i} \delta^{i} b^{\varepsilon}\left(x_{0}\right)^{i}\left[\left(D_{u} f_{a}\right)\left(D_{u} f\right)^{-1}\right]^{i+1},
\end{aligned}
$$

and $N_{1}, N_{2}$ are given in (2.23).

Proof. First, by the facts that

$$
D_{u} f+\delta b^{\varepsilon}\left(x_{0}\right) D_{u} f_{a}=\left[I+\delta b^{\varepsilon}\left(x_{0}\right)\left(D_{u} f_{a}\right)\left(D_{u} f\right)^{-1}\right]\left(D_{u} f\right),
$$

and $I+\delta b^{\varepsilon}\left(x_{0}\right)\left(D_{u} f_{a}\right)\left(D_{u} f\right)^{-1}$ is invertible for small $\delta>0$, we have

$$
\left(D_{u} f+\delta b^{\varepsilon}\left(x_{0}\right) D_{u} f_{a}\right)^{-1}=\left(D_{u} f\right)^{-1}\left(I+\delta b^{\varepsilon}\left(x_{0}\right)\left(D_{u} f_{a}\right)\left(D_{u} f\right)^{-1}\right)^{-1} .
$$

Next, since $b^{\varepsilon}\left(x_{0}\right)\left(D_{u} f_{a}\right)\left(D_{u} f\right)^{-1} \quad$ is uniformly bounded and $\delta^{i} b^{\varepsilon}\left(x_{0}\right)^{i}\left[\left(D_{u} f_{a}\right)\left(D_{u} f\right)^{-1}\right]^{i}$ approaches zero matrix as $\delta$ tends to zero,

$$
\left(I+\delta b^{\varepsilon}\left(x_{0}\right)\left(D_{u} f_{a}\right)\left(D_{u} f\right)^{-1}\right)^{-1}=I-\delta b^{\varepsilon}\left(x_{0}\right) J_{*},
$$

where $J_{*}$ is given in the lemma. Then, by applying (2.25) and (2.26) to (2.21), we obtain (2.24). We complete the proof. $\mathrm{C}$

Based on Lemma 2.1, we are ready to parametrize $u_{s}^{\varepsilon}$. According to the existence and uniqueness theorem of ordinary differential equations, the solution $\left(\eta^{\varepsilon}, u_{s}^{\varepsilon}\right)$ of (2.20) is a perturbation of the integral curve of $\left(1, R_{0}\left(\eta^{\varepsilon}, u_{s}^{\varepsilon}\right)\right)$, which starts at $\left(a_{L}, u_{1}\right)$. More precisely, $u_{s}^{\varepsilon}$ in $D_{M}^{\varepsilon}$ can be parametrized as

$$
\begin{aligned}
u_{s}^{\varepsilon}\left(\eta_{0} ; x_{0}, t_{0}, u_{1}\right)= & u_{1}+\eta_{0} R_{0}\left(a_{L}, u_{1}\right)+\frac{\eta_{0}^{2}}{2}\left(R_{0} \cdot \nabla R_{0}\right)\left(a_{L}, u_{1}\right) \\
& +\eta_{0} \delta K_{0}+O\left(\delta\left|\eta_{0}\right|^{2}+\left|\eta_{0}\right|^{3}\right),
\end{aligned}
$$

where

$$
K_{0}:=\left(J_{1}+J_{2}+\widetilde{L}\right),
$$

which depends on $a_{L}, u_{1}, a_{t}\left(x_{0}, t_{0}\right), a_{t x}\left(x_{0}, t_{0}\right)$ and $a_{t t}\left(x_{0}, t_{0}\right)$. Note that, by $\left(\mathrm{A}_{2}\right)$ and Lemma 2.1, the system in (2.20) has no equilibrium in $D_{M}^{\varepsilon}$ for sufficiently small $\delta>0$. Hence, the total variation of $u_{s}^{\varepsilon}$ in $D_{M}^{\varepsilon}$ can be controlled due to the monotonicity of $\eta^{\varepsilon}$. Also, $\eta_{0}$ equals to the total variation of $\bar{a}^{\varepsilon}$.

By the previous analysis we have the following theorem.

THEOREM 2.2. For the PRP (2.9), there exists a smooth parameter of states that can be connected to $u_{L}$ on the right by a smooth standing wave given by (2.20).

For the time-independent source, the standing waves are scale invariant and can be re-scaled into discontinuities. We then have

DeFINITION 2.3. An admissible solution $u_{s}$ is called the standing wave discontinuity of $\left(P_{0}\right)$ if $u_{s}$ is the $\varepsilon \downarrow 0$ limit of smooth standing waves $\left\{u_{s}^{\varepsilon}\right\}$.

To give a description of our generalized Riemann solver for $\left(P_{0}\right)$, we look for 2 intermediate states $u_{1}$ and $u_{2}$ such that $u_{L}, u_{1}, u_{2}, u_{R}$ are separated by shocks, rarefaction waves or the standing wave discontinuity, which is given in the following: 
Definition 2.4. Given $\left(x_{0}, t_{0}\right) \in \mathbb{R}^{+} \times[0, \infty)$ and $\kappa, \delta>0$ sufficiently small, we say that $u(x, t)$ is the generalized Riemann solver for $\left(P_{0}\right)$ if the following conditions hold:

(a) there exists two states $\left(a_{L}, u_{1}\right),\left(a_{R}, u_{2}\right)$ which satisfy $u_{2}=u_{s}\left(\eta_{0} ; x_{0}, t_{0}, u_{1}\right)$ for the wave strength $\eta_{0}:=a_{R}-a_{L}$;

(b) in region $\left[x_{0}-\kappa, x_{0}\right) \times\left[t_{0}, t_{0}+\delta\right], u(x, t)$ coincides with the 1-wave to the homogeneous PRP (2.9) with initial values $a_{L}, u_{L}, u_{1}$; while in the region $\left(x_{0}, x_{0}-\kappa\right] \times\left[t_{0}, t_{0}+\delta\right], u(x, t)$ coincides with the 2-wave to (2.9) with initial values $a_{R}, u_{2}, u_{R}$.

The next theorem establishes existence and uniqueness for such Riemann solver.

TheOrem 2.5. Given $\left(x_{0}, t_{0}\right) \in \mathbb{R}^{+} \times[0, \infty)$. Assume that conditions $\left(A_{1}\right)-\left(A_{3}\right)$ hold and that $u_{L}, u_{R} \in \Omega$ with $\left|u_{L}-u_{R}\right|$ sufficiently small. Then there exists a neighborhood $N \subset \Omega$ such that if $u_{L}, u_{R} \in N$, then there exists a unique Riemann solver of $\left(P_{0}\right)$ centered at $\left(x_{0}, t_{0}\right)$ in the sense of Definition 2.4.

Proof. In the previous analysis, we augment the system in (2.9) by adding $\bar{a}_{t}^{\varepsilon}=0$. So, let $U^{\varepsilon}:=\left(\bar{a}^{\varepsilon}, u^{\varepsilon}\right)^{T}$ and define

$$
R_{0}^{*}:=\left(1, R_{0}\right)^{T}, \quad R_{1}^{*}:=\left(0, R_{1}\right)^{T}, \quad R_{2}^{*}:=\left(0, R_{2}\right)^{T},
$$

and

$$
K_{i}^{*}:=\left(0, K_{i}\right)^{T}, \quad H_{i}^{*}\left(U^{\varepsilon}, \Lambda^{i}\right):=R_{i}^{*}\left(U^{\varepsilon}\right)+\delta K_{i}^{*}\left(\Lambda^{i}\right), \quad i=0,1,2,
$$

where $K_{1}, K_{2}$ are given in $(2.11), K_{0}$ is given in (2.28), and the parameter $\Lambda^{i}$ describes the point at which $K_{i}^{*}$ (or $K_{i}$ ) is evaluated. According to (2.15) and (2.27), for any constant state $u^{\varepsilon} \in \Omega$, there is a set of $C^{2}$-mappings $\left\{\widetilde{T}_{i}^{\delta}\right\}_{i=0}^{2}$ such that $\widetilde{T}_{i}^{\delta}\left(\sigma_{i} ; U^{\varepsilon}\right)$, $i=1,2$, can be connected to $U^{\varepsilon}$ on the right by either an $i$-shock or an $i$-rarefaction wave with the wave strength $\sigma_{i}$, and $\widetilde{T}_{0}^{\delta}\left(\sigma_{0} ; U^{\varepsilon}, x_{0}, t_{0}\right)$ can be connected to $U^{\varepsilon}$ on the right by a standing wave (zero-wave) of strength $\sigma_{0}$. Define the composite mapping

$$
\widetilde{T}^{\delta}\left(\sigma ; U^{\varepsilon}\right):=\widetilde{T}_{2}^{\delta}\left(\sigma_{2} ; \widetilde{T}_{0}^{\delta}\left(\sigma_{0} ; \widetilde{T}_{1}^{\delta}\left(\sigma_{1} ; U^{\varepsilon}\right), x_{0}, t_{0}\right)\right),
$$

where $\sigma:=\left(\sigma_{1}, \sigma_{0}, \sigma_{2}\right)$. By direct calculation we obtain

$$
\widetilde{T}^{\delta}\left(\sigma ; U_{L}\right)=U_{L}+\sum_{j=0}^{2} \sigma_{j} H_{j}^{*}\left(U_{L}, \Lambda_{L}^{j}\right)+O\left(\delta|\sigma|^{2}\right)+O\left(|\sigma|^{2}\right),
$$

where $U_{L}:=\left(a_{L}, u_{L}\right), \quad \Lambda_{L}^{1}=\Lambda_{L}^{2}=\left(a_{L}, b_{L}, u_{L}\right)$ and $\Lambda_{L}^{0}=$ $\left(U_{L}, a_{t}\left(x_{0}, t_{0}\right), a_{t x}\left(x_{0}, t_{0}\right), a_{t t}\left(x_{0}, t_{0}\right)\right)$. It is sufficient to show that, for every small $\delta>0$, there exists some $\eta:=\left(\eta_{1}, \eta_{0}, \eta_{2}\right)$ such that

$$
\widetilde{T}^{\delta}\left(\eta ; U_{L}\right)=U_{R}
$$

where $U_{R}:=\left(a_{R}, u_{R}\right)$.

Define

$$
T^{\delta}\left(\sigma ; U^{\varepsilon}\right):=\widetilde{T}^{\delta}\left(\sigma ; U^{\varepsilon}\right)-U^{\varepsilon}
$$

Then we have $T^{\delta}\left(0,0,0 ; U_{L}\right)=0$. In addition, the Jacobian matrix $D_{\sigma} T^{\delta}$ at $\sigma=$ $(0,0,0)$ can be calculated by

$$
\begin{aligned}
D_{\sigma} T^{\delta}\left(0,0,0 ; U_{L}\right) & =\left[H_{1}^{*}\left(U_{L}, \Lambda_{L}^{1}\right), H_{0}^{*}\left(U_{L}, \Lambda_{L}^{0}\right), H_{2}^{*}\left(U_{L}, \Lambda_{L}^{2}\right)\right] \\
& =R^{*}\left(U_{L}\right)+\delta K^{*}\left(\Lambda_{L}\right)
\end{aligned}
$$


where

$$
R^{*}:=\left[R_{1}^{*}, R_{0}^{*}, R_{2}^{*}\right], \quad K^{*}\left(\Lambda_{L}\right):=\left[K_{1}^{*}\left(\Lambda_{L}^{1}\right), K_{0}^{*}\left(\Lambda_{L}^{0}\right), K_{2}^{*}\left(\Lambda_{L}^{2}\right)\right]
$$

and $R_{1}^{*}, R_{0}^{*}$ and $R_{2}^{*}$ are given in (2.29). Since $\left\{R_{0}^{*}, R_{1}^{*}, R_{2}^{*}\right\}$ is linearly independent for all $u \in \Omega$, there exists a sufficiently small $\delta^{*}>0$ such that

$$
\operatorname{det}\left(D_{\sigma} T^{\delta}\left(0,0,0 ; U_{L}\right)\right)=\operatorname{det}\left(R^{*}\left(U_{L}\right)+\delta K^{*}\left(\Lambda_{L}\right)\right) \neq 0
$$

for any $\delta \in\left(0, \delta^{*}\right)$. Therefore, by the inverse function theorem, there exists a unique $\eta=\left(\eta_{1}, \eta_{0}, \eta_{2}\right)$ such that $(2.33)$ holds. The above argument is independent of $\varepsilon$. By taking the limit $\varepsilon \rightarrow 0$ and using the standing wave discontinuity as the zero-wave, we complete the proof.

In the rest of this section, we estimate the residual of approximate solution of (2.9) on $D_{\kappa \delta}:=\left[x_{0}-\kappa, x_{0}+\kappa\right] \times\left[t_{0}, t_{0}+\delta\right]$. Given $\phi \in C_{c}^{1}(E)$, the residual $R_{\phi}$ is defined by

$$
R_{\phi}\left(\bar{a}^{\varepsilon}, u^{\varepsilon}, D_{\kappa \delta}\right):=\iint_{D_{\kappa \delta}} u^{\varepsilon} \phi_{t}+f\left(\bar{a}^{\varepsilon}, u^{\varepsilon}\right) \phi_{x}+\left(\bar{a}^{\varepsilon}\right)^{\prime} g\left(\bar{a}^{\varepsilon}, \bar{a}^{\prime}, b^{\varepsilon}, u^{\varepsilon}\right) \phi d x d t .
$$

In Section 5, we will see that the estimate of $R_{\phi}$ is crucial to obtain the consistency of the GGS.

THEOREM 2.6. Let $u^{\varepsilon}$ be the approximate solution to (2.9) constructed by the modified Lax method for $0<\varepsilon \ll 1$. Then

$$
\begin{aligned}
R_{\phi}\left(\bar{a}^{\varepsilon}, u^{\varepsilon}, D_{\kappa \delta}\right)= & \int_{x_{0}-\kappa}^{x_{0}+\kappa} u^{\varepsilon}\left(x, t_{0}+\delta\right) \phi\left(x, t_{0}+\delta\right) d x-\int_{x_{0}-\kappa}^{x_{0}+\kappa} u^{\varepsilon}\left(x, t_{0}\right) \phi\left(x, t_{0}\right) d x \\
& +\int_{t_{0}}^{t_{0}+\delta}\left(f+\delta b_{R} f_{a}\right)\left(a_{R}, u^{\varepsilon}\left(x_{0}+\kappa, t\right)\right) \phi\left(x_{0}+\kappa, t\right) d t \\
& -\int_{t_{0}}^{t_{0}+\delta}\left(f+\delta b_{L} f_{a}\right)\left(a_{L}, u^{\varepsilon}\left(x_{0}-\kappa, t\right)\right) \phi\left(x_{0}-\kappa, t\right) d t \\
& +O\left(\kappa^{3}\right)+O\left(\kappa^{2}\right)\|\phi\|_{\infty}\left(\text { osc. }\left\{\eta^{\varepsilon} \text { in } D_{\kappa \delta}\right\}+\text { osc. }\left\{a_{t}\left(\cdot, t_{0}\right) \text { in } D_{\kappa \delta}\right\}\right) \\
& +O(\kappa) \varepsilon\|\phi\|_{\infty} \cdot \text { osc. }\left\{u^{\varepsilon} \text { in } D_{\kappa \delta}\right\},
\end{aligned}
$$

where osc. $\{u\}$ denotes the oscillation of $u$ and $\phi \in C_{c}^{1}(E)$.

Proof. First, by the construction of $u^{\varepsilon}$, it is easy to see that

$$
R_{\phi}\left(\bar{a}^{\varepsilon}, u^{\varepsilon}, D_{\kappa \delta}\right)=R_{\phi}\left(a_{L}, u^{\varepsilon}, D_{L}^{\varepsilon}\right)+R_{\phi}\left(\eta^{\varepsilon}, u_{s}^{\varepsilon}, D_{M}^{\varepsilon}\right)+R_{\phi}\left(a_{R}, u^{\varepsilon}, D_{R}^{\varepsilon}\right),
$$

where $D_{L}^{\varepsilon}, D_{M}^{\varepsilon}$ and $D_{R}^{\varepsilon}$ are given in (2.13). Next, we estimate $R_{\phi}$ in $D_{L}^{\varepsilon}$ and $D_{R}^{\varepsilon}$, respectively. Applying the divergence theorem to $R_{\phi}$ together with $b^{\varepsilon}=b_{L}$ and the 
Rankine-Hugoniot condition (2.17) for shock, we obtain

$$
\begin{aligned}
& R_{\phi}\left(a_{L}, u^{\varepsilon}, D_{L}^{\varepsilon}\right) \\
& =\iint_{D_{L}^{\varepsilon}}\left\{\left(u^{\varepsilon} \phi\right)_{t}+\left(\left(f+\delta b_{L} f_{a}\right)\left(a_{L}, u^{\varepsilon}\right) \phi\right)_{x}\right\} d x d t-\iint_{D_{L}^{\varepsilon}} \delta b_{L} f_{a}\left(a_{L}, u^{\varepsilon}\right) \phi_{x} d x d t \\
& =\int_{x_{0}-\kappa}^{x_{0}-\varepsilon \kappa} u^{\varepsilon}\left(x, t_{0}+\delta\right) \phi\left(x, t_{0}+\delta\right) d x-\int_{x_{0}-\kappa}^{x_{0}-\varepsilon \kappa} u^{\varepsilon}\left(x, t_{0}\right) \phi\left(x, t_{0}\right) d x \\
& \quad+\int_{t_{0}}^{t_{0}+\delta}\left(f+\delta b_{L} f_{a}\right)\left(a_{L}, u^{\varepsilon}\left(x_{0}-\varepsilon \kappa, t\right)\right) \phi\left(x_{0}-\varepsilon \kappa, t\right) d t
\end{aligned}
$$

$$
-\int_{t_{0}}^{t_{0}+\delta}\left(f+\delta b_{L} f_{a}\right)\left(a_{L}, u^{\varepsilon}\left(x_{0}-\kappa, t\right)\right) \phi\left(x_{0}-\kappa, t\right) d t+O\left(\kappa^{3}\right) .
$$

Similarly, we have

$$
\begin{aligned}
R_{\phi}\left(a_{R}, u^{\varepsilon}, D_{R}^{\varepsilon}\right)= & \int_{x_{0}+\varepsilon \kappa}^{x_{0}+\kappa} u^{\varepsilon}\left(x, t_{0}+\delta\right) \phi\left(x, t_{0}+\delta\right) d x-\int_{x_{0}+\varepsilon \kappa}^{x_{0}+\kappa} u^{\varepsilon}\left(x, t_{0}\right) \phi\left(x, t_{0}\right) d x \\
& -\int_{t_{0}}^{t_{0}+\delta}\left(f+\delta b_{R} f_{a}\right)\left(a_{R}, u^{\varepsilon}\left(x_{0}+\varepsilon \kappa, t\right)\right) \phi\left(x_{0}+\varepsilon \kappa, t\right) d t \\
& +\int_{t_{0}}^{t_{0}+\delta}\left(f+\delta b_{R} f_{a}\right)\left(a_{R}, u^{\varepsilon}\left(x_{0}+\kappa, t\right)\right) \phi\left(x_{0}+\kappa, t\right) d t+O\left(\kappa^{3}\right) .
\end{aligned}
$$

Finally, we calculate $R_{\phi}\left(\eta^{\varepsilon}, u_{s}^{\varepsilon}, D_{M}^{\varepsilon}\right)$. According to (2.19) and applying integration by parts to $R_{\phi}$, we obtain

$$
\begin{aligned}
R_{\phi}\left(\eta^{\varepsilon}, u_{s}^{\varepsilon}, D_{M}^{\varepsilon}\right)= & \int_{x_{0}-\varepsilon \kappa}^{x_{0}+\varepsilon \kappa} u_{s}^{\varepsilon}\left(x, t_{0}+\delta\right) \phi\left(x, t_{0}+\delta\right) d x-\int_{x_{0}-\varepsilon \kappa}^{x_{0}+\varepsilon \kappa} u^{\varepsilon}\left(x, t_{0}\right) \phi\left(x, t_{0}\right) d x \\
& +\int_{t_{0}}^{t_{0}+\delta}\left(f+\delta b\left(x_{0}\right) f_{a}\right)\left(a_{R}, u^{\varepsilon}\left(x_{0}+\varepsilon \kappa, t\right)\right) \phi\left(x_{0}+\varepsilon \kappa, t\right) d t \\
& -\int_{t_{0}}^{t_{0}+\delta}\left(f+\delta b\left(x_{0}\right) f_{a}\right)\left(a_{L}, u^{\varepsilon}\left(x_{0}-\varepsilon \kappa, t\right)\right) \phi\left(x_{0}-\varepsilon \kappa, t\right) d t \\
& +E_{1}\left(\eta^{\varepsilon}, u_{s}^{\varepsilon},\left(x_{0}, t_{0}\right)\right)+E_{0}\left(u_{s}^{\varepsilon},\left(x_{0}, t_{0}\right)\right),
\end{aligned}
$$

where

$$
\begin{aligned}
& E_{1}\left(\eta^{\varepsilon}, u_{s}^{\varepsilon},\left(x_{0}, t_{0}\right)\right):=-\iint_{D_{\varepsilon}} \delta b\left(x_{0}\right) f_{a}\left(\eta^{\varepsilon}, u_{s}^{\varepsilon}\right) \phi_{x} d x d t \\
&-\iint_{D_{\varepsilon}}\left(\eta^{\varepsilon}\right)^{\prime} \delta\left[b\left(x_{0}\right) g_{a}+b^{\prime}\left(x_{0}\right) g_{a_{x}}+c\left(x_{0}\right) g_{a_{t}}\right] \phi d x d t \\
&-\iint_{D_{\varepsilon}} \delta b^{\prime}\left(x_{0}\right)\left(g-f_{a}\right) \phi d x d t \\
& E_{0}\left(u_{s}^{\varepsilon},\left(x_{0}, t_{0}\right)\right):=\int_{x_{0}-\varepsilon \kappa}^{x_{0}+\varepsilon \kappa}\left(u^{\varepsilon}\left(x, t_{0}\right)-u_{s}^{\varepsilon}\left(x, t_{0}^{+}\right)\right) \phi\left(x, t_{0}\right) d x .
\end{aligned}
$$


The terms $E_{0}$ and $E_{1}$ can be estimated by

$$
\begin{aligned}
E_{1}\left(\eta^{\varepsilon}, \psi_{s}^{\varepsilon},\left(x_{0}, t_{0}\right)\right) & =O\left(\kappa^{3}\right)+O\left(\kappa^{2}\right)\|\phi\|_{\infty} \cdot \text { osc. }\left\{\eta^{\varepsilon} \text { in } D_{\kappa \delta}\right\} . \\
E_{0}\left(u_{s}^{\varepsilon},\left(x_{0}, t_{0}\right)\right) & =O(\kappa) \varepsilon\|\phi\|_{\infty} \cdot \text { osc. }\left\{u^{\varepsilon} \text { in } D_{\kappa \delta}\right\} .
\end{aligned}
$$

In addition, we see that

$$
\begin{aligned}
& \int_{t_{0}}^{t_{0}+\delta} \delta\left(b\left(x_{0}\right)-b_{R}\right) f_{a}\left(a_{R}, u^{\varepsilon}\left(x_{0}+\varepsilon \kappa, t\right)\right) \phi\left(x_{0}+\varepsilon \kappa, t\right) d t \\
& \quad-\int_{t_{0}}^{t_{0}+\delta} \delta\left(b\left(x_{0}\right)-b_{L}\right) f_{a}\left(a_{L}, u^{\varepsilon}\left(x_{0}-\varepsilon \kappa, t\right)\right) \phi\left(x_{0}-\varepsilon \kappa, t\right) d t \\
& =O\left(\kappa^{2}\right)\|\phi\|_{\infty} \cdot \text { osc. }\left\{a_{t}\left(\cdot, t_{0}\right) \text { in } D_{\kappa \delta}\right\} .
\end{aligned}
$$

Therefore, by (2.39)-(2.45), we obtain (2.38). The proof is complete.

3. Generalized boundary Riemann solver and its error estimate. In this section we study the following boundary Riemann problem near the boundary:

$$
\left\{\begin{array}{lc}
u_{t}+f(a, u)_{x}=a_{x} g\left(a, a_{x}, a_{t}, u\right), & (x, t) \in(0, \kappa) \times\left(t_{0}, t_{0}+\delta\right), \\
(a, u)\left(x, t_{0}\right)=\left(a_{R}, u_{R}\right), & 0<x<\kappa, \\
\left(a, u_{1}\right)(0, t)=\left(a_{B}, u_{1 B}\right), & t_{0}<t<t_{0}+\delta,
\end{array}\right.
$$

where $0<\kappa, \delta \ll 1$, and $\left(a_{R}, u_{R}\right)$ and $\left(a_{B}, u_{1 B}\right)$ are two constant states. By the same analysis used in $(2.2)-(2.8)$, we consider the corresponding perturbed boundary Riemann problem $(P B R P)$ for $0<\varepsilon \ll 1$ :

$$
\left(P_{\varepsilon}^{B}\right):\left\{\begin{array}{l}
\begin{array}{l}
u_{t}^{\varepsilon}+\left[\left(f+\delta b^{\varepsilon}(x) f_{a}\right)\left(\bar{a}^{\varepsilon}, u^{\varepsilon}\right)\right]_{x}=\left(\bar{a}^{\varepsilon}\right)^{\prime}\left[g+\delta\left(b^{\varepsilon}(x) g_{a}+\left(b^{\varepsilon}\right)^{\prime}(x) g_{a_{x}}\right.\right. \\
\left.\left.\quad+c(x) g_{a_{t}}\right)\right]+\delta\left(b^{\varepsilon}\right)^{\prime}(x) g,
\end{array} \\
u^{\varepsilon}\left(x, t_{0}\right)= \begin{cases}\psi_{B}^{\varepsilon}(x), & 0 \leq x \leq \varepsilon \kappa, \\
u_{R}, & \varepsilon \kappa<\leq \kappa, \\
\left(a, u_{1}\right)(0, t)=\left(a_{B}, u_{1 B}\right), & t_{0} \leq t<t_{0}+\delta,\end{cases}
\end{array}\right.
$$

where $g, g_{a}, g_{a_{x}}$ and $g_{a_{t}}$ are all evaluated at $\left(\bar{a}^{\varepsilon}, \bar{a}^{\prime}, b^{\varepsilon}, u^{\varepsilon}\right)$ with $\bar{a}^{\prime}=a_{x}\left(x, t_{0}\right), c(x):=$ $a_{t t}\left(x, t_{0}\right)$,

$$
\left(\bar{a}^{\varepsilon}(x), b^{\varepsilon}(x)\right):= \begin{cases}\left(\eta_{B}^{\varepsilon}(x), a_{t}\left(x, t_{0}\right)\right), & 0 \leq x \leq \varepsilon \kappa \\ \left(a_{R}, b_{R}\right), & \varepsilon \kappa<x \leq \kappa\end{cases}
$$

$\eta_{B}^{\varepsilon}(x)$ is a monotone function connecting $a_{B}$ at $x=0$ and $a_{R}$ at $x=\varepsilon \kappa$, respectively, and $\psi_{B}^{\varepsilon}(x)$ is a monotone function connecting boundary data $\left(u_{1 B}, u_{2 B}\right)$ at $x=0$ and $u_{R}$ at $x=\varepsilon \kappa$, respectively. It is remarked that $u_{2 B}$ will be decided by the modified Lax method. The limiting perturbed boundary Riemann problem, which is denoted by $\left(P_{0}^{B}\right)$, is defined by taking the limit $\varepsilon \rightarrow 0$ to $\left(P_{\varepsilon}^{B}\right)$. Our objective is to extend the results in Section 2 to $\left(P_{\varepsilon}^{B}\right)$ and $\left(P_{0}^{B}\right)$. Then, in view of Definition 2.4, a generalized Riemann solution to $\left(P_{0}^{B}\right)$ can be given by

$$
u(x, t):=\lim _{\varepsilon \rightarrow 0} u^{\varepsilon}(x, t),
$$

where $u^{\varepsilon}(x, t)$ is the approximate solution to $\left(P_{\varepsilon}^{B}\right)$.

We recall that the second characteristic field of $(3.2)$ is genuinely nonlinear. In the region $\left\{\varepsilon \kappa<x<\kappa, t_{0} \leq t<t_{0}+\delta\right\}$, a elementary wave curve is adopted on 
account of the absence of the source term. For given $U_{2}:=\left(a_{R}, u_{2}\right)$ and a sufficiently small parameter $\sigma_{2}$, there exists a $C^{2}$ mapping $\widetilde{T}_{2}^{\delta}$ such that

$$
\widetilde{T}_{2}^{\delta}\left(\sigma_{2} ; U_{2}\right)=\left(a_{R}, u_{2}+\sigma_{2} R_{2}\left(U_{2}\right)+\sigma_{2} \delta K_{2}\left(\Lambda_{2}^{2}\right)+O\left(\left|\sigma_{2}\right|^{2}\right)\right)=U_{R}
$$

where $U_{R}:=\left(a_{R}, u_{R}\right), R_{2}$ and $K_{2}$ are given in (2.11), and $\Lambda_{2}^{2}:=\left(a_{R}, b_{R}, u_{2}\right)$. In other words, $U_{R}$ can be connected by a 2 -wave curve starting at $U_{2}$ with the (signed) wave strength $\sigma_{2}$. For the zero characteristic field, we construct a time-independent approximate solution. Followed by the results in Section 2 , for given $U_{B}:=\left(a_{B}, u_{1 B}, u_{2 B}\right)$ nearby $U_{2}$, there exists a mapping $\widetilde{T}_{0}^{\delta}$ such that

$$
\widetilde{T}_{0}^{\delta}\left(\sigma_{0} ; U_{B}, t_{0}\right)=\left(a_{B}, u_{B}+\sigma_{0} R_{0}\left(U_{B}\right)+\sigma_{0} \delta K_{0}\left(\Lambda_{B}^{0}\right)+O\left(\left|\sigma_{0}\right|^{2}\right)\right)=U_{2},
$$

where $\sigma_{0}=a_{R}-a_{B}, \quad K_{0}$ is given in (2.28), and $\Lambda_{B}^{0}:=$ $\left(a_{B}, a_{t}\left(0, t_{0}\right), a_{t x}\left(0, t_{0}\right), a_{t t}\left(0, t_{0}\right), u_{B}\right)$, that is, $U_{2}$ can be connected by a zerowave curve starting at $U_{B}$ with the (signed) wave strength $\sigma_{0}$. Here $\widetilde{T}_{0}^{\delta}$ is at least a $C^{2}$ mapping of $\sigma_{0}$ and $U_{B}$, and $\sigma_{0}$ equals to the variation of $\bar{a}^{\varepsilon}$ near the boundary. Define the composite $\widetilde{T}_{B}$ of $\widetilde{T}_{2}^{\delta}$ and $\widetilde{T}_{0}^{\delta}$ by

$$
\widetilde{T}_{B}\left(\sigma_{0}, \sigma_{2} ; U_{B}\right):=\widetilde{T}_{2}^{\delta}\left(\sigma_{2} ; \widetilde{T}_{0}^{\delta}\left(\sigma_{0} ; U_{B}, t_{0}\right)\right) .
$$

Then, by (3.4)-(3.6), we obtain

$$
\widetilde{T}_{B}\left(\sigma_{0}, \sigma_{2} ; U_{B}\right)=U_{B}+\sum_{j=0,2} \sigma_{j} H_{j}^{*}\left(U_{B}, \Lambda_{B}^{j}\right)+O\left(\left|\sigma_{0}\right|+\left|\sigma_{2}\right|\right)^{2}=U_{R}
$$

where $H_{j}^{*}, j=0,2$, are given in $(2.30)$, and $\Lambda_{B}^{2}:=\left(a_{B}, b_{B}, u_{B}\right)$ with $b_{B}:=a_{t}\left(0, t_{0}\right)$. Clearly, by $(3.7)$, we have $\widetilde{T}_{B}\left(0,0 ; U_{B}\right)=U_{B}$, and

$$
\left.\frac{\partial \widetilde{T}_{B}}{\partial \sigma_{0}}\right|_{\left(0,0 ; U_{B}\right)}=R_{0}^{*}\left(U_{B}\right)+\delta K_{0}^{*}\left(\Lambda_{B}^{0}\right),\left.\quad \frac{\partial \widetilde{T}_{B}}{\partial \sigma_{2}}\right|_{\left(0,0 ; U_{B}\right)}=R_{2}^{*}\left(U_{B}\right)+\delta K_{2}^{*}\left(\Lambda_{B}^{2}\right) .
$$

Now, we consider the mapping $T_{B}$ given by

$$
T_{B}\left(\sigma_{0}, \sigma_{2}\right):=\left[\widetilde{T}_{B}\left(\sigma_{0}, \sigma_{2} ; U_{B}\right)\right]_{1,2}-\left[U_{B}\right]_{1,2},
$$

where $[U]_{1,2}$ denotes the 2 -vector consisting of the first two components of $U$. Then, by $(3.7)-(3.9)$, we have $T_{B}(0,0)=(0,0)$ and the Jacobian matrix

$$
D T_{B}(0,0)=\left[\begin{array}{cc}
1 & 0 \\
{\left[R_{0}\left(U_{B}\right)+\delta K_{0}\left(\Lambda_{B}^{0}\right)\right]_{1}} & {\left[R_{2}\left(U_{B}\right)+\delta K_{2}\left(\Lambda_{B}^{2}\right)\right]_{1}}
\end{array}\right] .
$$

To apply Lax's method, we need the following lemma.

Lemma 3.1. The matrix $D T_{B}(0,0)$ in $(3.10)$ is non-singular provided that $\left(A_{2}\right)$ holds.

Proof. Suppose that $D T_{B}(0,0)$ is singular. It means $\left[R_{2}\left(U_{B}\right)+\delta K_{2}\left(\Lambda_{B}^{2}\right)\right]_{1}=0$ and so

$$
\lambda_{2}\left(U_{B}\right)\left[R_{2}\left(U_{B}\right)+\delta K_{2}\left(\Lambda_{B}^{2}\right)\right]_{1}=0
$$


Moreover, since $R_{2}+\delta K_{2}$ is a nonzero right eigenvector of $D_{u} f^{\delta}$, we have $\left[R_{2}\left(U_{B}\right)+\right.$ $\left.\delta K_{2}\left(\Lambda_{B}^{2}\right)\right]_{2} \neq 0$ and

$$
D_{u} f^{\delta}\left(b_{B}, U_{B}\right) \cdot\left(R_{2}\left(U_{B}\right)+\delta K_{2}\left(\Lambda_{B}^{2}\right)\right)=\lambda_{2}\left(U_{B}\right)\left(R_{2}\left(U_{B}\right)+\delta K_{2}\left(\Lambda_{B}^{2}\right)\right) .
$$

It follows by (3.11) and (3.12) that

$$
\frac{\partial}{\partial u_{2}}\left(f_{1}+\delta b_{B} f_{1 a}\right)\left(U_{B}\right)=\frac{\partial}{\partial u_{2}} f_{1}^{\delta}\left(b_{B}, U_{B}\right)=\frac{\lambda_{2}\left(U_{B}\right)\left[R_{2}\left(U_{B}\right)+\delta K_{2}\left(\Lambda_{B}^{2}\right)\right]_{1}}{\left[R_{2}\left(U_{B}\right)+\delta K_{2}\left(\Lambda_{B}^{2}\right)\right]_{2}}=0,
$$

which violates the condition $\left(A_{2}\right)$ for sufficiently small $\delta$. The proof is complete.

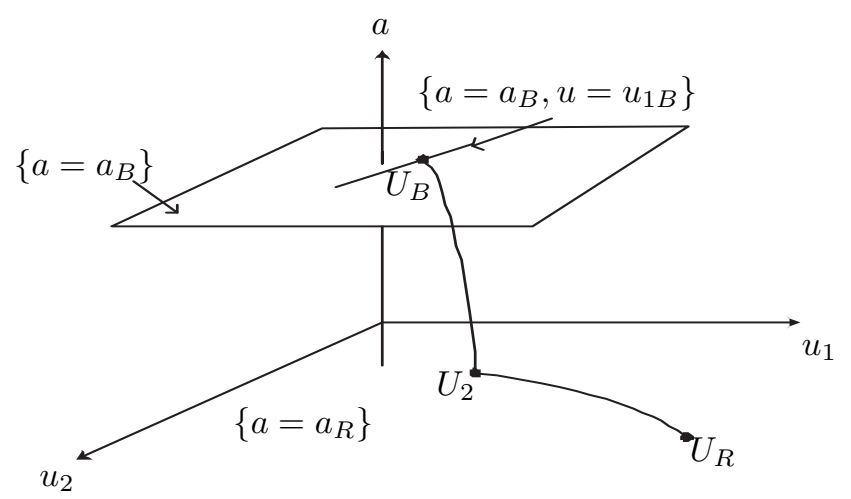

Fig. 1. The approximate solution to the PBRP in the phase plane.

We are now ready to prove the existence of approximate solutions to (3.2). First, by Lemma 3.1 and the inverse function theorem, there exist two neighborhoods $N_{0}$ of $(0,0)$ and $N_{B}$ of $\left[U_{B}\right]_{1,2}$ such that $T_{B}$ is a diffeomorphism from $N_{0}$ to $N_{B}$. It means that we can solve $T_{B}$ for $\sigma_{0}$ and $\sigma_{2}$ in terms of $U_{R}$ and $U_{B}$ when $\left|\left(a_{B}, u_{1 B}\right)-\left(a_{R}, u_{1 R}\right)\right|$ is sufficiently small. Next, we determine $u_{2 B}$ when $\left[U_{R}\right]_{1,2}=\left(a_{R}, u_{1 R}\right) \in N_{B}$ is given. In view of $(3.7)$, we have

$$
\left[\widetilde{T}_{B}\left(\sigma_{0}, \sigma_{2} ; U_{B}\right)\right]_{3}=u_{2 B}+\sum_{j=0,2} \sigma_{j}\left[H_{j}^{*}\left(U_{B}, \Lambda_{B}^{j}\right)\right]_{3}+O\left(\left|\sigma_{0}\right|+\left|\sigma_{2}\right|\right)^{2} .
$$

By (3.13), we see that

$$
\frac{d\left[\widetilde{T}_{B}\left(\sigma_{0}, \sigma_{2} ; U_{B}\right)\right]_{3}}{d u_{2 B}}=1+\sum_{j=0,2} \sigma_{j} \frac{d\left[H_{j}^{*}\left(U_{B}, \Lambda_{B}^{j}\right)\right]_{3}}{d u_{2 B}}+O\left(\left|\sigma_{0}\right|+\left|\sigma_{2}\right|\right)^{2} .
$$

In particular, we obtain $d\left[\widetilde{T}_{B}\left(0,0 ; U_{B}\right)\right]_{3} / d u_{2 B}=1$. Therefore, by the inverse function theorem, there exists a unique $u_{2 B}$ which is nearby $u_{2 R}$ and satisfies (3.7) for sufficiently small $\sigma_{0}$ and $\sigma_{2}$. By the analysis above, we have the following theorem regarding to the existence and uniqueness of solutions to $\left(P_{\varepsilon}^{B}\right)$.

TheOREM 3.2. Consider $\left(P_{\varepsilon}^{B}\right)$ given in (3.2) with $u_{R} \in \Omega$ and sufficiently small $\left|\left(a_{B}, u_{1 B}\right)-\left(a_{R}, u_{1 R}\right)\right|$. Then, under conditions $\left(A_{1}\right)-\left(A_{3}\right)$, there exists a unique approximate solution $u^{\varepsilon}(x, t)$ to $\left(P_{\varepsilon}^{B}\right)$ obtained by the modified Lax method. The approximate solution $u^{\varepsilon}(x, t)$ consists of at most three constant states separated by a 2-wave issued from $(\varepsilon, 0)$, and a smooth standing wave in $0 \leq x \leq \varepsilon$. Moreover, there exists a unique $u_{2 B}$ such that $u^{\varepsilon}(0, t)=\left(u_{1 B}, u_{2 B}\right)$ for $t_{0}<t<t_{0}+\delta$, see Fig. 1 . 
The structure of $u^{\varepsilon}(x, t)$ depends on the choice of $\bar{a}^{\varepsilon}$. However, the wave curves for $\left(\bar{a}^{\varepsilon}, u^{\varepsilon}\right)$ are determined uniquely on the phase plane. It means that the states in $u^{\varepsilon}(x, t)$ are independent of $\bar{a}^{\varepsilon}$ and $\varepsilon$. By letting $\varepsilon \rightarrow 0$ in $u^{\varepsilon}(x, t)$, we generate a generalized boundary Riemann solver for $\left(P_{0}^{B}\right)$.

COROLlary 3.3. Consider the limiting case of perturbed boundary Riemann problem $\left(P_{0}^{B}\right)$. Then, under the same hypothesis as in Theorem 3.2, $\left(P_{0}^{B}\right)$ admits a unique boundary Riemann solver $u(x, t):=\lim _{\varepsilon \rightarrow 0} u^{\varepsilon}(x, t)$ consisting of at most three constant states separated by the 2-wave and the standing wave discontinuity. Furthermore, there exists a unique constant $u_{2 B}$ such that $u(0, t)=\left(u_{1 B}, u_{2 B}\right)$ for $t_{0}<t<t_{0}+\delta$.

TheOREm 3.4. Let $\phi \in C_{c}^{1}(E)$ and $D_{\kappa \delta}^{B}:=[0, \kappa] \times\left[t_{0}, t_{0}+\delta\right]$. Let $u^{\varepsilon}$ be the approximate solution to $\left(P_{\varepsilon}^{B}\right)$ constructed by the modified Lax method. Then

$$
\begin{aligned}
R_{\phi}\left(\bar{a}^{\varepsilon}, u^{\varepsilon}, D_{\kappa \delta}^{B}\right)= & \int_{0}^{\kappa} u^{\varepsilon}\left(x, t_{0}+\delta\right) \phi\left(x, t_{0}+\delta\right) d x-\int_{0}^{\kappa} u^{\varepsilon}\left(x, t_{0}\right) \phi\left(x, t_{0}\right) d x \\
& +\int_{t_{0}}^{t_{0}+\delta}\left(f+\delta b_{R} f_{a}\right)\left(a_{R}, u^{\varepsilon}(\kappa, t)\right) \phi(\kappa, t) d t \\
& -\int_{t_{0}}^{t_{0}+\delta}\left(f+\delta b_{B} f_{a}\right)\left(a_{B}, u^{\varepsilon}(0, t)\right) \phi(0, t) d t \\
& +O\left(\kappa^{3}\right)+O\left(\kappa^{2}\right)\left(\text { osc. }\left\{\eta^{\varepsilon} \text { in } D_{\kappa \delta}^{B}\right\}+\text { osc. }\left\{a_{t}\left(\cdot, t_{0}\right) \text { in } D_{\kappa \delta}^{B}\right\}\right) \\
& +O(\kappa) \varepsilon\|\phi\|_{\infty} \cdot \text { osc. }\left\{u^{\varepsilon} \text { in } D_{\kappa \delta}^{B}\right\} .
\end{aligned}
$$

4. Generalized Glimm scheme and its stability. In this section we introduce the generalized Glimm scheme (GGS) for (1.1), (1.2) and establish its stability. Compared with the initial-boundary value problem, the analysis for the initial value problem is simpler. Therefore, we focus primarily on the case of (1.2).

To describe the scheme for (1.2), let $x_{k}:=k \Delta x, t_{i}:=i \Delta t, k, i=0,1,2, \ldots$, and divide the first quadrant plane into the time strips of length $\Delta t=O(\Delta x)$ :

$$
T_{i}:=\left\{(x, t) \mid 0 \leq x<\infty, t_{i} \leq t<t_{i+1}\right\}, \quad i=0,1,2, \ldots .
$$

Here $\Delta x$ and $\Delta t$ satisfy the generalized Courant-Friedrichs-Levy condition:

$$
\frac{\Delta x}{\Delta t}>(1-\varepsilon)^{-1} \sup \left\{\left|\lambda_{i}(a, u)\right|: i=1,2\right\}, \quad 0<\varepsilon \ll 1 .
$$

We also define

$$
\widetilde{\Delta x}:=(1+\varepsilon)^{-1} \Delta x
$$

When introducing the GGS, we impose (4.1) to ensure that waves emanating simultaneously from points $\left\{\left(\varepsilon \widetilde{\Delta x}, t_{i}\right),\left(x_{k} \pm \varepsilon \widetilde{\Delta x}, t_{i}\right)\right\}$ do not interact on a time interval of length $\Delta t$. Here we use the non-staggered grid points for the computational domain. In each perturbed Riemann or boundary Riemann problem, we augment the equation $\bar{a}_{t}^{\varepsilon}=0$ to the system. Hence, we need to provide the initial and boundary data for $a$ and $u$ in each time step. Let $U:=(a, u)$. Following the arguments in Sections 2 and 3 , we approximate initial data $U_{0}(x)$, boundary data $\left(a(0, t), u_{1 B}(t)\right)$ and $a_{t}(x, 0)$ as follows. 
$(\mathrm{I})_{0}$ The initial data $U_{0}^{\varepsilon}(x)$ on $[0, \infty)$ and the boundary data $\left(a_{B}^{0}, u_{1 B}^{0}\right)$ on $\left[0, t_{1}\right)$ are decided by

$$
\begin{aligned}
& U_{0}^{\varepsilon}(x)=\left(a_{0}^{\varepsilon}(x), u_{0}^{\varepsilon}(x)\right)= \begin{cases}\Psi_{B}^{0}(x), & 0 \leq x \leq \varepsilon \widetilde{\Delta x}, \\
U_{1}^{0}, & \varepsilon \widetilde{\Delta x}<x \leq x_{1}, \\
U_{k-1}^{0}, & x_{k-1} \leq x<x_{k}-\varepsilon \widetilde{\Delta x}, \quad k=2,4,6, \ldots, \\
\Psi_{k}^{0}(x), & \left|x-x_{k}\right| \leq \varepsilon \widetilde{\Delta x} \\
U_{k+1}^{0}, & x_{k}+\varepsilon \widetilde{\Delta x}<x \leq x_{k+1},\end{cases} \\
& \left(a_{B}^{0}(t), u_{1 B}^{0}(t)\right)=\left(a(0, \Delta t / 2), u_{1 B}(\Delta t / 2)\right), \quad 0 \leq t<t_{1},
\end{aligned}
$$

where $U_{k-1}^{0}=\left(a_{k-1}^{0}, u_{k-1}^{0}\right):=U_{0}\left(x_{k-1}\right)=\left(a\left(x_{k-1}, 0\right), u_{0}\left(x_{k-1}, 0\right)\right), \Psi_{k}^{0}(x):=$ $\left(\eta_{\varepsilon}^{0}(x), \psi_{k}^{0}(x)\right)$ is a smooth monotone function which connects $U_{k-1}^{0}$ at $x=x_{k}-\varepsilon \widetilde{\Delta x}$ and $U_{k+1}^{0}$ at $x=x_{k}+\varepsilon \widetilde{\Delta x}$, respectively, and $\Psi_{B}^{0}(x):=\left(\eta_{B}^{0}(x), \psi_{B}^{0}(x)\right)$ is a smooth monotone function connecting $\left(a_{B}^{0}, u_{1 B}^{0}, u_{2 B}^{0}\right)$ at $x=0$ and $U_{1}^{0}$ at $x=\varepsilon \widetilde{\Delta x}$, respectively. Here, $u_{2 B}^{0}$ is decided by the modified Lax method. Moreover, the approximation $b_{0}^{\varepsilon}(x)$ to $a_{t}(x, 0)$ is decided by

$$
b_{0}^{\varepsilon}(x)= \begin{cases}a_{t, 1}^{0}, & \varepsilon \widetilde{\Delta x}<x \leq x_{1} \\ a_{t, k-1}^{0}, & x_{k-1} \leq x<x_{k}-\varepsilon \widetilde{\Delta x} \\ a_{t}(x, 0), & 0 \leq x \leq \varepsilon \widetilde{\Delta x},\left|x-x_{k}\right| \leq \varepsilon \widetilde{\Delta x}, \quad k=2,4,6, \ldots \\ a_{t, k+1}^{0}, & x_{k}+\varepsilon \widetilde{\Delta x}<x \leq x_{k+1}\end{cases}
$$

where $a_{t, k-1}^{0}:=a_{t}\left(x_{k-1}, 0\right)$ for $k=2,4,6, \ldots$ We then solve the PBRP and the PRPs centered at $\left(x_{k}, 0\right), k=2,4,6, \ldots$, by the modified Lax method. One generates an approximate solution in the zero time strip $T_{0}$.

Let $u^{\varepsilon, i-1}(x, t), 0<\varepsilon \ll 1$, denote the approximate solution in the time strip $T_{i-1}$. For the ith time step, we choose the initial data $u_{i}^{\varepsilon}(x)$ by random choice along with the boundary data $\left(a_{B}^{i}, u_{1 B}^{i}\right), a_{i}^{\varepsilon}(x)$ and $b_{i}^{\varepsilon}(x)$ as follows.

$(\mathrm{I})_{i}$ The initial data $U_{i}^{\varepsilon}(x)$ on $[0, \infty)$ and the boundary data $\left(a_{B}^{i}, u_{1 B}^{i}\right)$ on $\left[t_{i}, t_{i+1}\right)$ are decided by

$$
\begin{aligned}
& U_{i}^{\varepsilon}(x)=\left(a_{i}^{\varepsilon}, u_{i}^{\varepsilon}\right)(x)=\left\{\begin{array}{l}
\Psi_{B}^{i}(x), \quad 0 \leq x \leq \varepsilon \widetilde{\Delta x}, \\
U_{1}^{i}, \quad \varepsilon \widetilde{\tilde{\Delta x}<x \leq x_{1},} \\
U_{k-1}^{i}, \quad x_{k-1} \leq x<x_{k}-\varepsilon \widetilde{\Delta x}, \quad k=2,4,6, \ldots, \\
\Psi_{k}^{i}(x), \quad\left|x-x_{k}\right| \leq \varepsilon \widetilde{\Delta x}, \\
U_{k+1}^{i}, \quad x_{k}+\varepsilon x<x \leq x_{k+1},
\end{array}\right. \\
& \left(a_{B}^{i}(t), u_{1 B}^{i}(t)\right)=\left(a\left(0, t_{i}+\Delta t / 2\right), u_{1 B}\left(t_{i}+\Delta t / 2\right)\right), \quad t_{i} \leq t<t_{i+1},
\end{aligned}
$$

where $U_{k-1}^{i}=\left(a_{k-1}^{i}, u_{k-1}^{i}\right):=\left(a\left(x_{k-1}, t_{i}\right), u^{\varepsilon, i-1}\left(x_{k-1}+\theta_{i} \widetilde{\Delta x}, t_{i}^{-}\right)\right), \theta_{i} \in(-1,1)$ is a random number, $\Psi_{k}^{i}(x):=\left(\eta_{\varepsilon}^{i}(x), \psi_{k}^{i}(x)\right)$ is a smooth monotone function connecting $U_{k-1}^{i}$ at $x=x_{k}-\varepsilon \widetilde{\Delta x}$ and $U_{k+1}^{i}$ at $x=x_{k}+\varepsilon \widetilde{\Delta x}$, respectively, and $\Psi_{B}^{i}(x)=$ $\left(\eta_{B}^{i}(x), \psi_{B}^{i}(x)\right)$ is a smooth monotone function connecting $\left(a_{B}^{i}, u_{1 B}^{i}, u_{2 B}^{i}\right)$ at $x=0$ and $U_{1}^{i}$ at $x=\varepsilon \widetilde{\Delta x}$, respectively, where $u_{2 B}^{i}$ is decided by the modified Lax method. 
In addition, $b_{i}^{\varepsilon}(x)$ is decided by

$$
b_{i}^{\varepsilon}(x)=\left\{\begin{array}{ll}
a_{t, 1}^{i}, & \varepsilon \widetilde{\Delta x}<x \leq x_{1} \\
a_{t, k-1}^{i}, & x_{k-1} \leq x<x_{k}-\varepsilon \widetilde{\Delta x} \\
a_{t}\left(x, t_{i}\right), & 0 \leq x \leq \varepsilon \widetilde{\Delta x},\left|x-x_{k}\right| \leq \varepsilon \widetilde{\Delta x}, \\
a_{t, k+1}^{i}, & x_{k}+\varepsilon \widetilde{\Delta x}<x \leq x_{k+1}
\end{array} \quad k=2,4,6, \ldots\right.
$$

where $a_{t, k-1}^{i}:=a_{t}\left(x_{k-1}, t_{i}\right)$ for $k=2,4,6, \ldots$ Again, by the results in Sections 2 and 3 , we obtain an approximate solution in $T_{i}$. Applying this process to each time step, there exists an approximate solution $u_{\theta, \Delta x}^{\varepsilon}$ constructed by the GGS with the random sequence $\theta:=\left(\theta_{1}, \theta_{2}, \ldots\right)$ in $(-1,1)$ and $0<\varepsilon \ll 1$. By taking the $\varepsilon \downarrow 0$ limit of $\left\{u_{\theta, \Delta x}^{\varepsilon}\right\}$, we generate the generalized Riemann solution, denoted by $u_{\theta, \Delta x}$, of (1.2).

Here we emphasize that, in every ith time step, the choice of $a_{i}^{\varepsilon}$, also denoted by $a_{\Delta x}^{\varepsilon}$, only depend on the values of $a$ at $\left(x_{k-1}, t_{i}\right), k=2,4,6, \ldots$, but not on the ones in the previous time step. Moreover, the random points $\left\{\left(x_{k-1}+\theta_{i} \widetilde{\Delta x}, t_{i}^{-}\right): k=\right.$ $2,4,6, \ldots, i \in \mathbb{N}\}$ are chosen outside the domains of standing waves to preserve the total variation of $u_{\theta, \Delta x}^{\varepsilon}$. This will not affect the equi-distributed property of random sequences as $\varepsilon$ approaches 0 .

To obtain the compactness of approximate solutions, the stability of the GGS is required. Due to the effect of $a(x, t)$, the interaction estimates in [9] are needed to be generalized.

We begin with the interaction of waves away from the boundary. Here we adopt the notations in [31]. Given $\left(x_{k}, t_{i}\right) \in \mathbb{R}^{+} \times[0, \infty), U:=(a, u)$ and $V:=(\bar{a}, v)$. Let

$$
(U, V):=\left[\left(U_{0}, U_{1}, U_{2}, U_{3}\right) /\left(\sigma_{0}, \sigma_{1}, \sigma_{2}\right)\right]
$$

denote the approximate solution to (2.9). That is, $(U, V)$ consists of four constant states, $U=U_{0}, U_{1}, U_{2}, U_{3}=V$, separated by the $j$-wave curve $\widetilde{T}_{j}^{\delta}\left(\sigma_{j} ; U_{j}\right)=U_{j+1}$ for $j=0,1,2$. On account of $(2.30)$ and $(2.36),\left[H_{0}^{*}, H_{1}^{*}, H_{2}^{*}\right]$ is nonsingular and its inverse satisfies

$$
\left[H_{0}^{*}, H_{1}^{*}, H_{2}^{*}\right]^{-1}\left(U, \Lambda ; x_{k}, t_{i}\right)=\left(\mathrm{R}^{*}\right)^{-1}(U)-(\Delta t)\left(\mathrm{R}^{*}\right)^{-1}(U) \mathrm{K}^{*}(\Lambda)\left(\mathrm{R}^{*}\right)^{-1}(U)+O(\Delta x)^{2} \text {, }
$$

where $\mathrm{R}^{*}$ and $\mathrm{K}^{*}$ are given in (2.35). Therefore, the function $V=T^{\delta}\left(\sigma ; U, x_{k}, t_{i}\right)$ can be inverted on a small neighborhood of $(0,0,0)$ to give

$$
\sigma=\left(\sigma_{0}, \sigma_{1}, \sigma_{2}\right)=\Theta\left(V ; U, x_{k}, t_{i}\right)
$$

where $\Theta$ is smooth in $\left(V ; U, x_{k}, t_{i}\right), \Theta\left(U ; U, x_{k}, t_{i}\right)=(0,0,0)$ and

$$
D_{V} \Theta\left(U ; U, x_{k}, t_{i}\right)=\left(\mathrm{R}^{*}\right)^{-1}(U)-(\Delta t)\left(\mathrm{R}^{*}\right)^{-1}(U) \mathrm{K}^{*}(\Lambda)\left(\mathrm{R}^{*}\right)^{-1}(U)+O(\Delta x)^{2}
$$

The function $\Theta$ solves the PRP (2.9) centered at $\left(x_{k}, t_{i}\right)$, that is, for fixed $u \in \Omega$, $a \in \mathbb{R}$ and for $v \in \Omega, \bar{a} \in \mathbb{R}$ with $|v-u|$ small, the jump discontinuity $\{u, v\}$ is resolved into $U=U_{0}, U_{1}, U_{2}$ and $U_{3}=V$ such that $U_{j+1}$ is connected to $U_{j}$, on the right, by a $j$-wave of strength $\sigma_{j}=\Theta_{j}\left(V ; U, x_{k}, t_{i}\right)$. Similarly, chose $V^{\prime}, U^{\prime} \in \Omega$ so that $\left(U^{\prime}, V^{\prime}\right)$ can be expressed as

$$
\left(U^{\prime}, V^{\prime}\right):=\left[\left(U_{0}^{\prime}, U_{1}^{\prime}, U_{2}^{\prime}, U_{3}^{\prime}\right) /\left(\sigma_{0}^{\prime}, \sigma_{1}^{\prime}, \sigma_{2}^{\prime}\right)\right],
$$


where $\sigma^{\prime}:=\left(\sigma_{0}^{\prime}, \sigma_{1}^{\prime}, \sigma_{2}^{\prime}\right)=\Theta\left(V^{\prime} ; U^{\prime}, x_{k}^{\prime}, t_{i}^{\prime}\right)$. Then, by (4.6) and the fact that $|V-U|=$ $O(1)\left|\Theta\left(V ; U, x_{k}, t_{i}\right)\right|=O(1)|\sigma|$, we obtain

$$
\begin{aligned}
& \sigma^{\prime}-\sigma \\
&=\int_{0}^{1} \frac{d}{d \xi} \Theta\left(U^{\prime}+\xi\left(V^{\prime}-U^{\prime}\right) ; U^{\prime}, x_{k}^{\prime}, t_{i}^{\prime}\right) d \xi-\int_{0}^{1} \frac{d}{d \xi} \Theta\left(U+\xi(V-U) ; U, x_{k}, t_{i}\right) d \xi \\
&=\int_{0}^{1}\left(D_{V} \Theta\left(U^{\prime}+\xi\left(V^{\prime}-U^{\prime}\right) ; U^{\prime}, x_{k}^{\prime}, t_{i}^{\prime}\right)-D_{V} \Theta\left(U+\xi(V-U) ; U, x_{k}, t_{i}\right)\right)(V-U) d \xi \\
&+\int_{0}^{1} D_{V} \Theta\left(U^{\prime}+\xi\left(V^{\prime}-U^{\prime}\right) ; U^{\prime}, x_{k}^{\prime}, t_{i}^{\prime}\right)\left(\left(V^{\prime}-U^{\prime}\right)-(V-U)\right) d \xi \\
&= O(1)|\sigma|\left(\left|V^{\prime}-V\right|+\left|U^{\prime}-U\right|+(\Delta t)\left|x_{k}^{\prime}-x_{k}\right|+(\Delta t)\left|t_{i}^{\prime}-t_{i}\right|+(\Delta x)^{2}\right) \\
&+O(1)\left|\left(V^{\prime}-U^{\prime}\right)-(V-U)\right| .
\end{aligned}
$$

We say that an $i$-wave and a $j$-wave are approaching if either (i) the wave on the left possesses larger speed or (ii) $i=j$ and at least one of them is a shock. In any case, two zero-waves are never approaching each other. Let $\theta=\left(\theta_{0}, \theta_{1}, \theta_{2}, \ldots\right)$, $\theta_{0}=0$, be an equi-distributed sequence of random numbers in $(-1,1)$. The points $P_{k, i}:=\left(x_{k}+\theta_{i} \widetilde{\Delta x}, t_{i}\right), P_{0, i}:=\left(0,\left(i+\frac{1}{2}\right) \Delta t\right), i=0,1,2, \ldots, k=1,3,5, \ldots$, are called the mesh points. We can connect those neighboring mesh points to get a set of diamond regions. In addition, the diamond regions near the boundary $x=0$ are triangles, see Fig. 3. An unbounded piecewise linear curve $I$ is a mesh curve if $I$ lies on the boundaries of those diamond regions. Hence if $I$ is a mesh curve, then $I$ divides the first quadrant plane into $I^{+}$and $I^{-}$parts such that $I^{-}$contains $t=0$. It reminds us that every mesh curve contains some unbounded portion of the boundary. The mesh curves $I_{2}>I_{1}$ if every point of $I_{2}$ is either on $I_{1}$ or contained in $I_{1}^{+}$. If $I_{2}>I_{1}$ and if all mesh points on $I_{2}$ except one are also on $I_{1}$, then $I_{2}$ is called an immediate successor of $I_{1}$.

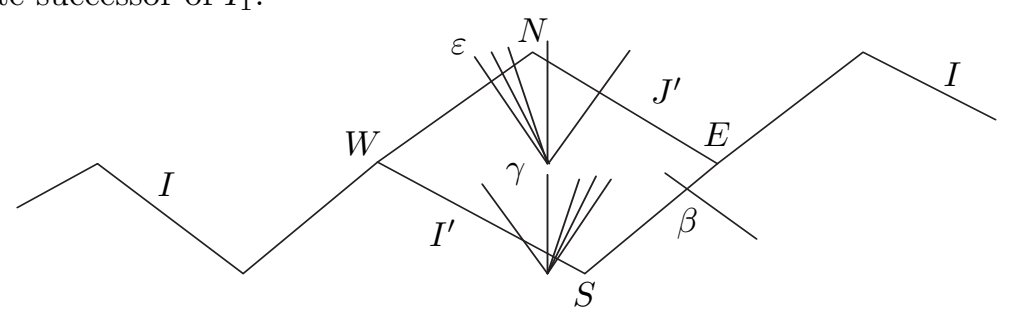

Fig. 2. A interior diamond region and in-coming and out-going waves

Let $\Delta$ be a diamond region centered at $\left(x_{m}, t_{n+1}\right), m \in\{2,4,6, \ldots\}$, and suppose that its vertices (mesh points) $N:=\left(x_{m-1}+\theta_{n+2} \widetilde{\Delta x}, t_{n+2}\right), E:=\left(x_{m+1}+\right.$ $\left.\theta_{n+1} \widetilde{\Delta x}, t_{n+1}\right), S:=\left(x_{m+1}+\theta_{n} \widetilde{\Delta x}, t_{n}\right)$ and $W:=\left(x_{m-1}+\theta_{n+1} \widetilde{\Delta x}, t_{n+1}\right)$, where $\left\{\theta_{n}, \theta_{n+1}, \theta_{n+2}\right\} \subset(-1,1)$ are the random numbers obtained by GGS, see Fig. 2. Moreover, let $\partial \Delta^{-}$stand for the lower boundary of $\Delta$ connecting $W, S$ and $E$, and $\partial \Delta^{+}$for the upper one connecting $W, N$ and $E$. We call waves across $\partial \Delta^{-}$and $\partial \Delta^{+}$ respectively the in-coming waves and the out-going waves of $\Delta$. Notice that there is at most one standing wave from the in-coming waves of $\Delta$. We have the following interaction estimate away from the boundary.

TheOREM 4.1. (Interior wave interaction estimate) Let $U_{L}^{\prime}:=\left(a_{L}^{\prime}, u_{L}\right), U_{R}^{\prime}:=$ 
$\left(a_{R}^{\prime}, u_{R}\right), U_{L}:=\left(a_{L}, u_{L}\right), U_{R}:=\left(a_{R}, u_{R}\right)$, and let $U_{M}:=\left(a_{M}, u_{M}\right)$ be a constant state near $U_{L}$ and $U_{R}$. Assume that

$$
\varepsilon=\Theta\left(U_{R}^{\prime} ; U_{L}^{\prime}, x_{m}, t_{n+1}\right)
$$

are the out-going waves of $\Delta$, and that

$$
\gamma=\Theta\left(U_{M} ; U_{L}, x_{m}, t_{n}\right), \quad \beta=\Theta\left(U_{R} ; U_{M}, x_{m+2}, t_{n}\right)
$$

are the in-coming waves of $\Delta$ with $\gamma_{0}$ across $\partial \Delta^{-}$, see Fig. 2. Then we have

$$
\begin{aligned}
\varepsilon= & \gamma+\beta+O(1) D(\gamma, \beta)+O(1)(|\gamma|+|\beta|)\left(\left|a_{t}\right|+\left|a_{x t}\right|\right)(\Delta t) \\
& +O(1)(\Delta x)(\Delta t)\left|a_{x t}\right|+\mathcal{C}(\Delta x,|\gamma|+|\beta|)
\end{aligned}
$$

as $|\gamma|+|\beta| \rightarrow 0$, where $a_{t}$ and $a_{x t}$ are evaluated at $\left(x_{m-1}, t_{n}\right), D(\gamma, \beta):=$ $\sum_{\text {Approaching }}\left|\gamma_{i}\right|\left|\beta_{j}\right|$ and $\mathcal{C}(\Delta x,|\gamma|+|\beta|)$ denotes the cubic terms of $\Delta x,|\gamma|$ and $|\beta|$.

Proof. We construct $\hat{\beta}=\Theta\left(U_{R} ; U_{M}, x_{m}, t_{n}\right)$ and $\hat{\varepsilon}=\Theta\left(U_{R} ; U_{L}, x_{m}, t_{n}\right)$. Then, by (4.8), we have

$$
\hat{\beta}=\beta+O(1)|\beta|(\Delta x)^{2} .
$$

In addition, by the results of $[9,12]$ and $(4.10)$, we yield

$$
\hat{\varepsilon}=\gamma+\hat{\beta}+O(1) D(\gamma, \hat{\beta})=\gamma+\beta+O(1) D(\gamma, \hat{\beta})+\mathcal{C}(\Delta x,|\beta|)
$$

as $|\gamma|+|\hat{\beta}| \rightarrow 0$, where $D(\gamma, \hat{\beta}):=\sum_{A p p}\left|\gamma_{i}\right|\left|\hat{\beta}_{j}\right|$ and $\mathcal{C}(\Delta x,|\beta|)$ denotes the cubic terms of $\Delta x$ and $|\beta|$. It remains to relate $\varepsilon$ to $\hat{\varepsilon}$ and $D(\gamma, \beta)$ to $D(\gamma, \hat{\beta})$, respectively. By (4.10), we obtain

$$
D(\gamma, \hat{\beta})=D(\gamma, \beta)+O(1)|\gamma||\beta|(\Delta x)^{2}
$$

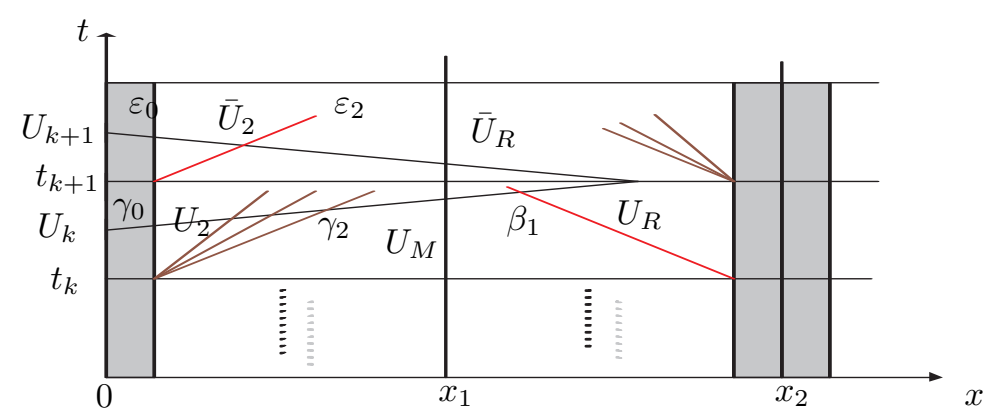

Fig. 3: Approximate solutions to the PRPs and PBRPs.

From (4.8), (4.11) and (4.12), it follows

$$
\begin{aligned}
\varepsilon= & \hat{\varepsilon}+O(1)|\hat{\varepsilon}|\left\{\left|\zeta_{L}\right|+\left|\zeta_{R}\right|+(\Delta x)^{2}\right\}+O(1)\left|\zeta_{R}-\zeta_{L}\right| \\
= & \gamma+\beta+O(1) D(\gamma, \beta)+O(1)(|\gamma|+|\beta|)\left(\left|\zeta_{L}\right|+\left|\zeta_{R}\right|\right)+O(1)\left|\zeta_{R}-\zeta_{L}\right| \\
& +\mathcal{C}(\Delta x,|\gamma|+|\beta|)
\end{aligned}
$$

as $|\gamma|+|\beta| \rightarrow 0$, where $\zeta_{R}:=a_{R}^{\prime}-a_{R}, \zeta_{L}:=a_{L}^{\prime}-a_{L}$. Since

$a_{R}^{\prime}=a\left(x_{m+1}, t_{n+1}\right), \quad a_{R}=a\left(x_{m+1}, t_{n}\right), \quad a_{L}^{\prime}=a\left(x_{m-1}, t_{n+1}\right), \quad a_{L}=a\left(x_{m-1}, t_{n}\right)$, 
so, by the Taylor expansion, we have

$$
\begin{aligned}
\zeta_{L} & =(\Delta t) a_{t}\left(x_{m-1}, t_{n}\right)+\frac{(\Delta t)^{2}}{2} a_{t t}\left(x_{m-1}, t_{n}\right)+O(\Delta x)^{3}, \\
\zeta_{R} & =(\Delta t) a_{x t}\left(x_{m-1}, t_{n}\right)+2(\Delta x)(\Delta t) a_{x t}\left(x_{m-1}, t_{n}\right)+O(\Delta x)^{3}, \\
\zeta_{R}-\zeta_{L} & =2(\Delta x)(\Delta t) a_{x t}\left(x_{m-1}, t_{n}\right)+O(\Delta x)^{3} .
\end{aligned}
$$

It follows by $(4.13)-(4.16)$ that $(4.9)$ is achieved. We complete the proof.

It remains to study the boundary wave interaction. Since $a$ is a function of $t$ on the boundary, the wave interaction estimate is more complicated than the one in [15]. However, we will show that the results in [15] can be extended to our problem. First, we recall the results in [15].

LEMmA 4.2. [15] (a) (Elementary wave interaction) Assume that

$$
W=\widetilde{T}_{j}^{\delta}\left(\beta_{j} ; \widetilde{T}_{i}^{\delta}\left(\gamma_{i} ; U\right)\right), \quad W^{\prime}=\widetilde{T}_{i}^{\delta}\left(\gamma_{i} ; \widetilde{T}_{j}^{\delta}\left(\beta_{j} ; U\right)\right)
$$

where $\widetilde{T}_{i}^{\delta}$ and $\widetilde{T}_{j}^{\delta}$ are given in (2.31). Then there exists a continuous function $\eta$ of $\gamma_{i}$ and $\beta_{j}$ such that

$$
W-W^{\prime}=\eta\left(\gamma_{i}, \beta_{j}\right) \gamma_{i} \beta_{j}
$$

(b) (Combining waves of the same family) Assume that

$$
V=\widetilde{T}_{i}^{\delta}\left(\gamma_{i} ; U\right), \quad W=\widetilde{T}_{i}^{\delta}\left(\beta_{i} ; V\right), \quad W^{\prime}=\widetilde{T}_{i}^{\delta}\left(\gamma_{i}+\beta_{i} ; U\right)
$$

Then there exists a continuous function $\zeta$ of $\gamma_{i}$ and $\beta_{i}$ such that

$$
W-W^{\prime}= \begin{cases}0, & \text { if } \gamma_{i} \text { and } \beta_{i} \text { are both rarefaction waves } \\ & \text { or both smooth standing waves } \\ \zeta\left(\gamma_{i}, \beta_{i}\right) \gamma_{i} \beta_{i}, & \text { otherwise. }\end{cases}
$$

By Lemma 4.2, we have the following theorem regarding to the boundary interaction estimate.

THEOREM 4.3. (Boundary wave interaction estimate) Let $U_{k}:=\left(a_{B}^{k}, u_{1 B}^{k}, u_{2 B}^{k}\right)$, $U_{R}:=\left(a_{R}^{k}, u_{R}\right), U_{k+1}:=\left(a_{B}^{k+1}, u_{1 B}^{k+1}, u_{2 B}^{k+1}\right)$ and $\bar{U}_{R}:=\left(a_{R}^{k+1}, u_{R}\right)$. Suppose that $\left(U_{k}, U_{M}\right):=\left[\left(U_{k}, U_{2}, U_{M}\right) /\left(\gamma_{0}, \gamma_{2}\right)\right]$ and $\left(U_{k+1}, \bar{U}_{R}\right):=\left[\left(U_{k+1}, \bar{U}_{2}, \bar{U}_{R}\right) /\left(\varepsilon_{0}, \varepsilon_{2}\right)\right]$ are the solutions to PBRPs on the $k$ th and the $(k+1)$ th time strips, respectively. Also, let $\left(U_{M}, U_{R}\right):=\left[\left(U_{M}, U_{R}\right) /\left(\beta_{1}\right)\right]$ be the 1-wave of the solution to PRP right next to $\left(U_{k}, U_{M}\right)$ on the kth time strip, see Fig. 3. Then there exists a constant $C$ such that for $j=0,2$,

$$
\begin{aligned}
\left|\varepsilon_{j}-\left(\gamma_{j}+\beta_{1}\right)\right| \leq & C\left(\sum_{A p p .}\left|\gamma_{i}\right|\left|\beta_{1}\right|+\left|\beta_{1}\right|+\left|v_{B}^{k+1}-v_{B}^{k}\right|+\left|a_{B}^{k+1}-a_{B}^{k}\right|+\left|a_{R}^{k+1}-a_{R}^{k}\right|\right) \\
& +O(\Delta x)^{2} .
\end{aligned}
$$

Proof. Let $\left(\bar{U}_{k}, \bar{U}_{R}\right):=\left[\left(\bar{U}_{k}, \bar{U}_{2}^{\prime}, \bar{U}_{R}\right) /\left(\alpha_{0}, \alpha_{2}\right)\right]$ be the approximate solution to the PBRP $\left[\bar{U}_{k}, \bar{U}_{R}\right]$, where $\bar{U}_{k}:=\left(a_{B}^{k}, v_{B}^{k}, \bar{w}_{B}^{k}\right)$ and $\bar{w}_{B}^{k}$ can be decided uniquely by the framework in Section 3. We emphasize that, due to the time-dependence of $a$, we 
solve the problem $\left[\bar{U}_{k}, \bar{U}_{R}\right]$ rather than $\left[\bar{U}_{k}, U_{R}\right]$ given in [15]. Then the terms of the LHS of (4.19) are bounded by

$$
\left|\varepsilon_{j}-\left(\gamma_{j}+\beta_{1}\right)\right| \leq\left|\varepsilon_{j}-\alpha_{j}\right|+\left|\alpha_{j}-\left(\gamma_{j}+\beta_{1}\right)\right|, \quad j=0,2 .
$$

We estimate $\left|\varepsilon_{j}-\alpha_{j}\right|$. In view of $T_{B}$ in (3.9), there exists a smooth function $\widetilde{\Theta}(V ; U, t)=\left(\widetilde{\Theta}_{0}(V ; U, t), \widetilde{\Theta}_{2}(V ; U, t)\right)$ such that

$$
\left(\varepsilon_{0}, \varepsilon_{2}\right)=\widetilde{\Theta}\left(\bar{U}_{R} ; U_{k+1}, t_{k+1}\right), \quad\left(\alpha_{0}, \alpha_{2}\right)=\widetilde{\Theta}\left(\bar{U}_{R} ; \bar{U}_{k}, t_{k}\right) .
$$

The function $\widetilde{\Theta}$ solves, for instance, the jump discontinuity $\left\{U_{k+1}, \bar{U}_{R}\right\}$ into $U_{k+1}$, $\bar{U}_{2}$ and $\bar{U}_{R}$ such that $\bar{U}_{2}$ is connected to $U_{k+1}$ on the right by a 0 -wave of strength $\varepsilon_{0}=\widetilde{\Theta}_{0}\left(\bar{U}_{R} ; U_{k+1}, t_{k+1}\right)$, and $\bar{U}_{R}$ is connected to $\bar{U}_{2}$ on the right by a 2 -wave of strength $\varepsilon_{2}=\widetilde{\Theta}_{2}\left(\bar{U}_{R} ; U_{k+1}, t_{k+1}\right)$. Applying patterns of similar calculations in (4.8) to (4.21), we obtain

$$
\left|\varepsilon_{j}-\alpha_{j}\right| \leq C\left(\left|v_{B}^{k+1}-v_{B}^{k}\right|+\left|a_{B}^{k+1}-a_{B}^{k}\right|\right)+O(\Delta x)^{2}, \quad j=0,2 .
$$

The term $\left|\alpha_{j}-\left(\gamma_{j}+\beta_{1}\right)\right|$ is now estimated as follows. We notice that the map

$$
U_{R}=\widetilde{T}_{i}^{\delta}\left(\tau_{i} ; U_{L}\right) \text { is smooth in }\left(\tau_{i}, U_{L}\right),
$$

where $U_{R}$ is the state which can be connected to $U_{L}$ on the right by an $i$-wave of strength $\tau_{i}$ (Section 3). We first study the case that $\beta_{1}$ interacts with $\gamma_{2}$ and $\gamma_{0}$ in order, see Fig. 4. By Lemma 4.2, the states $U_{1}^{\prime}, U_{M}^{\prime}$ and $U_{R}^{\prime}$ in Fig. 4 can be completely determined by the interaction of waves $\beta_{1}, \gamma_{0}$ and $\gamma_{2}$, respectively. Furthermore, following (4.17), (4.23) and the triangle inequality, we obtain

$$
\left|U_{R}^{\prime}-U_{R}\right| \leq C\left(\left|\gamma_{0} \beta_{1}\right|+\left|\gamma_{2} \beta_{1}\right|\right)
$$

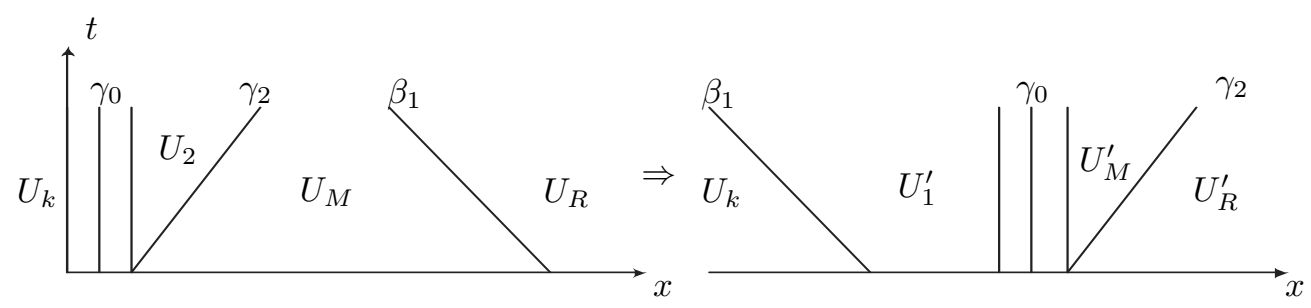

Fig. 4: Wave interaction with interchange of waves twice.

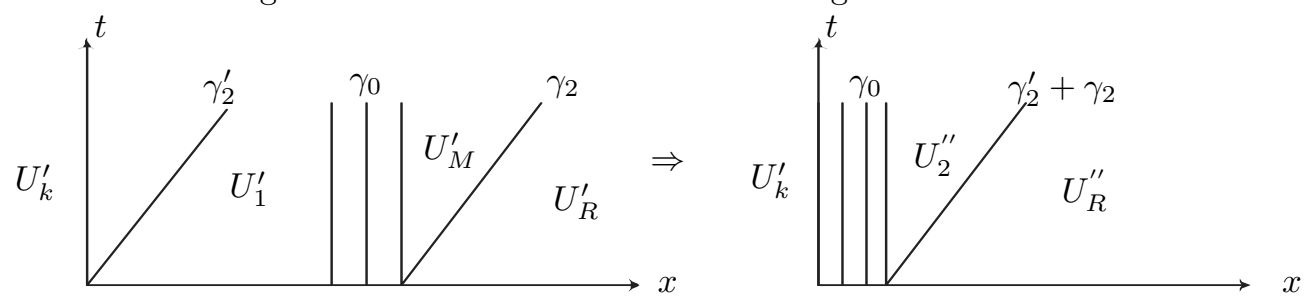

Fig. 5: Interchange and combination of waves.

where $U_{R}^{\prime}$ is connected to $U_{k}$ on the right by waves $\beta_{1}, \gamma_{0}$ and $\gamma_{2}$. On the other hand, it is easy to see that

$$
\left|\left[U_{1}^{\prime}\right]_{1,2}-\left(a_{B}^{k}, u_{1 B}^{k}\right)\right| \leq\left|U_{1}^{\prime}-U_{k}\right| \leq C\left|\beta_{1}\right|
$$


where $U_{1}^{\prime}:=\left(a_{B}^{k}, u_{1}^{\prime}, u_{2}^{\prime}\right)$ is connected to $U_{k}$ on the right by $\beta_{1}$, and $\left[U_{1}^{\prime}\right]_{1,2}$ stands for the first two components of $U_{1}^{\prime}$.

Next, we solve the PBRP $\left[\left(a_{B}^{k}, u_{1 B}^{k}, *\right), U_{1}^{\prime}\right]$, see Fig. 5. Again, by the results in Section 3, the solution to $\left[\left(a_{B}^{k}, u_{1 B}^{k}, *\right), U_{1}^{\prime}\right]$ exists and $*$ is decided uniquely. Let $u_{2 B}^{k \prime}=*$ and $U_{k}^{\prime}:=\left(a_{B}^{k}, u_{1 B}^{k}, u_{2 B}^{k \prime}\right)$, and let $\left(U_{k}^{\prime}, U_{1}^{\prime}\right):=\left[\left(U_{k}^{\prime}, U_{1}^{\prime}\right) /\left(\gamma_{2}^{\prime}\right)\right]$ be the solution to $\left[U_{k}^{\prime}, U_{1}^{\prime}\right]$. Then we estimate the case that $\gamma_{2}^{\prime}$ interchanges with $\gamma_{0}$ and then combines with $\gamma_{2}$, see Fig. 5. By the differentiability of $\widetilde{\Theta}$ and (4.25), it follows that $\gamma_{2}^{\prime}$ satisfies

$$
\left|\gamma_{2}^{\prime}\right| \leq C\left|\left(a_{B}^{k}, u_{1 B}^{k}\right)-\left[U_{1}^{\prime}\right]_{1,2}\right| \leq C\left|\beta_{1}\right| .
$$

By (4.17), (4.18), (4.23) and the triangle inequality, we obtain

$$
\left|U_{R}^{\prime \prime}-U_{R}^{\prime}\right| \leq C\left(\left|\gamma_{0} \gamma_{2}^{\prime}\right|+\left|\gamma_{2} \gamma_{2}^{\prime}\right|\right) \leq C\left(\left|\gamma_{2}^{\prime}\right|\right),
$$

where $U_{R}^{\prime \prime}$ is connected to $U_{k}^{\prime}$ on the right by waves $\gamma_{0}$ and $\gamma_{2}^{\prime}+\gamma_{2}$. Here we used the fact that wave strengths $\gamma_{0}$ and $\gamma_{2}$ are bounded. It follows by (4.26) and (4.27) that

$$
\left|U_{R}^{\prime \prime}-U_{R}^{\prime}\right| \leq C\left|\beta_{1}\right| \text {. }
$$

In view of $\left(\bar{U}_{k}, \bar{U}_{R}\right)=\left[\left(\bar{U}_{k}, \bar{U}_{2}^{\prime}, \bar{U}_{R}\right) /\left(\alpha_{0}, \alpha_{2}\right)\right]$, and by (4.24), (4.28) and the differentiability of $\widetilde{\Theta}$, we obtain that $\alpha_{j}, j=0,2$, satisfy

$$
\begin{aligned}
\left|\alpha_{j}-\left(\gamma_{j}^{\prime}+\gamma_{j}\right)\right| & \leq C\left|\left[\bar{U}_{R}\right]_{1,2}-\left[U_{R}^{\prime \prime}\right]_{1,2}\right| \leq C\left(\left|\bar{U}_{R}-U_{R}\right|+\left|U_{R}-U_{R}^{\prime}\right|+\left|U_{R}^{\prime}-U_{R}^{\prime \prime}\right|\right) \\
& \leq C\left(\left|a_{R}^{k+1}-a_{R}^{k}\right|+\left|\gamma_{0} \beta_{1}\right|+\left|\gamma_{2} \beta_{1}\right|+\left|\beta_{1}\right|\right)+O(\Delta x)^{2},
\end{aligned}
$$

where $\gamma_{0}^{\prime}:=0$. Finally, by (4.26) and (4.29), we have

$$
\left|\alpha_{j}-\left(\gamma_{j}+\beta_{1}\right)\right| \leq\left|\alpha_{j}-\left(\gamma_{j}^{\prime}+\gamma_{j}\right)\right|+\left|\gamma_{j}^{\prime}\right|+\left|\beta_{1}\right|
$$

Therefore, by (4.20), (4.22) and (4.30), we establish (4.19). The proof is complete. $\square$

Based on the estimates in Theorems 4.1 and 4.3 , we begin to establish the stability of the GGS. It is sufficient to show that the total variations of approximate solutions stay uniformly bounded in time and this can be accomplished by a globally nonincreasing Glimm functional introduced by Glimm [9]. Because of the presence of the boundary data, $\left|\beta_{1}\right|$ and $\left|a_{R}^{k+1}-a_{R}^{k}\right|$ in (4.19), we propose a slight modification of Glimm functional as follows.

$$
F(I):=L(I)+K Q(I),
$$

where $I$ is any mesh curve, and

$$
\begin{aligned}
L(I) & :=\sum\left\{\left|\gamma_{i}\right|: \gamma_{i} \operatorname{crosses} I\right\}+K_{1}\left(\left|\beta_{1}\right|+\sum_{k \in B(I)} l_{B}^{k}\right), \\
Q(I) & :=\sum\left\{\left|\gamma_{i}\right|\left|\gamma_{i^{\prime}}\right|: \gamma_{i}, \gamma_{i^{\prime}} \operatorname{cross} I \text { and approach }\right\}, \\
l_{B}^{k} & :=\left|u_{1 B}^{k+1}-u_{1 B}^{k}\right|+\left|a_{B}^{k+1}-a_{B}^{k}\right|+(\Delta x)\left(\left|a_{x B}^{k+1}\right|+\left|a_{t B}^{k+1}\right|+\left|a_{x B}^{k}\right|+\left|a_{t B}^{k}\right|\right) .
\end{aligned}
$$

Here, constants $K>1$ and $K_{1}>1$ will be decided later. $B(I):=\left\{k: P_{0, k}=\right.$ $\left.\left(0, t_{k}+\Delta t / 2\right) \in I\right\}, u_{1 B}^{k+1}:=u_{1}\left(P_{0, k+1}\right)$ and $a_{t B}^{k}:=a_{t}\left(P_{0, k}\right)$ etc. The term $\left|\beta_{1}\right|$ is involved in $L(I)$ when $\beta_{1}$ crosses $I$ and locates in some boundary triangle region, 
see Fig. 3. We notice that, in the initial value problem (1.1), we let $K_{1}=0$ in the functional $L$ since there is no wave-reflection on the boundary.

TheOREM 4.4. Let T.V. $\left\{U_{0}, u_{1 B}, a_{B}\right\}:=T . V .\left\{U_{0}\right\}+T . V .\left\{u_{1 B}\right\}+T . V .\{a(0, \cdot)\}$ and $\omega(E):=\left\|a_{t}\right\|_{L^{1}(E)}+\left\|a_{x t}\right\|_{L^{1}(E)}$, where $E=[0, \infty) \times[0, \infty)$. If T.V. $\left\{U_{0}, u_{1 B}, a_{B}\right\}$ and $\omega(E)$ are sufficiently small, then the approximate solution $u_{\theta, \Delta x}^{\varepsilon}$ is well-defined for $t>0$ and $0<\varepsilon \ll 1$.

Proof. Let $I$ and $J$ be two mesh curves and $J$ an immediate successor of $I$. First, suppose that $I$ and $J$ enclose an interior diamond region centered at $\left(x_{m}, t_{n+1}\right)$, see Fig. 2. Then, with the help of (4.9), we obtain

$L(J)-L(I) \leq O(1)\left\{D(\gamma, \beta)+(|\gamma|+|\beta|)\left(\left|a_{t}\right|+\left|a_{x t}\right|\right)(\Delta t)+(\Delta x)(\Delta t)\left|a_{x t}\right|\right\}$

$Q(J)-Q(I) \leq O(1) L(I)\left\{D(\gamma, \beta)+(|\gamma|+|\beta|)\left(\left|a_{t}\right|+\left|a_{x t}\right|\right)(\Delta t)+(\Delta x)(\Delta t)\left|a_{x t}\right|\right.$

$$
\left.+O(\Delta x)^{3}\right\}-D(\gamma, \beta)
$$

where $a_{t}$ and $a_{x t}$ are evaluated at $\left(x_{m-1}, t_{n}\right)$. It follows by $(4.31)-(4.33)$ that

$$
\begin{aligned}
F(J)-F(I) \leq & O(1)[1+K L(I)]\left\{D(\gamma, \beta)+(|\gamma|+|\beta|)\left(\left|a_{t}\right|+\left|a_{x t}\right|\right)(\Delta t)\right. \\
& \left.+(\Delta x)(\Delta t)\left|a_{x t}\right|+O(\Delta x)^{3}\right\}-K D(\gamma, \beta) .
\end{aligned}
$$

Next, if $J$ and $I$ enclose a triangle region containing the mesh point $P_{0, k}$, see Fig. 3 , then by $(4.31)$ we obtain

$$
\begin{aligned}
F(J)-F(I)= & \left|\varepsilon_{0}\right|+\left|\varepsilon_{2}\right|-\left|\gamma_{0}\right|-\left|\gamma_{2}\right|-\left|\beta_{1}\right|-K_{1}\left(\left|\beta_{1}\right|+l_{B}^{k}\right) \\
& +K\left(\sum_{A p p .}\left|\alpha_{i}\right|\left|\varepsilon_{j}\right|-\sum_{A p p .}\left|\alpha_{i}\right|\left|\gamma_{j}\right|-\sum_{A p p .}\left|\alpha_{i}\right|\left|\beta_{1}\right|\right)-K\left(\left|\gamma_{0} \beta_{1}\right|+\left|\gamma_{2} \beta_{1}\right|\right) .
\end{aligned}
$$

Here every summation above is taken over all approaching waves $\left\{\alpha_{i}\right\}$ on the right of $\beta_{1}$, in addition, $\left\{\alpha_{i}\right\}$ also crosses $I$. It follows from (4.35) and Theorem 4.3 that

$$
\begin{aligned}
F(J)-F(I) \leq & O(1) C\left(\left|\gamma_{0} \beta_{1}\right|+\left|\gamma_{2} \beta_{1}\right|+\left|\beta_{1}\right|+l_{B}^{k}\right)-K_{1}\left(\left|\beta_{1}\right|+l_{B}^{k}\right) \\
& +O(1) C K \sum\left|\alpha_{i}\right|\left(\left|\gamma_{0} \beta_{1}\right|+\left|\gamma_{2} \beta_{1}\right|+\left|\beta_{1}\right|+l_{B}^{k}\right) \\
& -K\left(\left|\gamma_{0} \beta_{1}\right|+\left|\gamma_{2} \beta_{1}\right|\right)+O(\Delta x)^{2} \\
\leq & \left(-K_{1}+O(1) C+O(1) C K \cdot F(I)\right)\left(\left|\beta_{1}\right|+l_{B}^{k}\right) \\
& +(-K+O(1) C+O(1) C K \cdot F(I))\left(\left|\gamma_{0} \beta_{1}\right|+\left|\gamma_{2} \beta_{1}\right|\right)+O(\Delta x)^{2} \\
\leq & \nu(\Delta x)^{2}
\end{aligned}
$$

for some positive constant $\nu$ provided that constants $K_{1}, K \geq O(1) 2 C$, and $K F(I) \leq$ 1 .

Now, let $J_{n}$ be the mesh curve that is located on the time strip $T_{n}$ for $x>0$ and includes the half-ray $t \geq t_{n}+\Delta t / 2$ for $x=0$. Summing up (4.34) for even $m$ together 
with (4.36), we have

$$
\begin{aligned}
F\left(J_{n+1}\right)-F\left(J_{n}\right)= & O(1)\left[1+K L\left(J_{n}\right)\right] Q\left(J_{n}\right)-K Q\left(J_{n}\right) \\
& +O(1)(\Delta t) \sum_{m}(|\gamma|+|\beta|)\left(C_{m}^{n}+\bar{C}_{m}^{n}\right) \\
& +O(1)(\Delta x)(\Delta t) \sum_{m}\left\|a_{x t}\right\|_{L^{\infty}\left(D_{n m}\right)}+O(\Delta x)^{2} \\
\leq & O(1)(\Delta t) L\left(J_{n}\right) \sup _{m}\left(C_{m}^{n}+\bar{C}_{m}^{n}\right) \\
& +O(1)(\Delta x)(\Delta t) \sum_{m}\left\|a_{x t}\right\|_{L^{\infty}\left(D_{n m}\right)}+O(\Delta x)^{2},
\end{aligned}
$$

provided that $K \geq O(1) 2 C$ and $K F\left(J_{n}\right) \leq 1$, where $D_{n m}:=\left[x_{m-1}, x_{m+1}\right] \times\left[t_{n}, t_{n+1}\right]$, and

$$
C_{m}^{n}:=\sup _{x_{m-1} \leqslant x \leqslant x_{m+1}}\left|a_{t}\left(x, t_{n}\right)\right|, \quad \bar{C}_{m}^{n}:=\sup _{x_{m-1} \leqslant x \leqslant x_{m+1}}\left|a_{x t}\left(x, t_{n}\right)\right| .
$$

Furthermore, if $a_{x}(0, t)$ and $a_{t}(0, t)$ are bounded, then for sufficiently small $T . V .\left\{U_{0}, u_{1 B}, a_{B}\right\}$ and $\Delta x$, we have

$$
\begin{aligned}
F\left(J_{0}\right) & \leq L\left(J_{0}\right)+K L\left(J_{0}\right)^{2}=\left(L\left(J_{0}\right)+K L\left(J_{0}\right)\right) L\left(J_{0}\right) \\
& \leq O(1)\left[1+K_{1} K \cdot T \cdot V \cdot\left\{U_{0}, u_{1 B}, a_{B}\right\}\right] K_{1} \cdot T . V \cdot\left\{U_{0}, u_{1 B}, a_{B}\right\} \\
& \leq O(1) 2 K_{1} \cdot T . V \cdot\left\{U_{0}, u_{1 B}, a_{B}\right\} .
\end{aligned}
$$

Therefore, by (4.37), (4.38) and by induction hypothesis, there exists a positive constant $M^{*}$ such that

$$
\begin{aligned}
L\left(J_{k}\right) \leq & F\left(J_{k}\right) \leq M^{*} \quad \text { for } k=0,1, \ldots, n, \\
F\left(J_{n+1}\right) \leq & F\left(J_{0}\right)+O(1)(\Delta t) M^{*} \sum_{k=0}^{n} \sup _{m}\left(C_{m}^{k}+\bar{C}_{m}^{k}\right) \\
& +O(1)(\Delta x)(\Delta t) \sum_{k=0}^{n} \sum_{m}\left\|a_{x t}\right\|_{L^{\infty}\left(D_{k m}\right)}+O(\Delta x) .
\end{aligned}
$$

From the assumption that the constant $\omega(E)$ is finite, we see that

$$
\begin{aligned}
& \lim _{\Delta x \rightarrow 0} \sum_{k=0}^{\infty} \sup _{m}\left(C_{m}^{k}+\bar{C}_{m}^{k}\right)(\Delta t)=\omega(E), \\
& \lim _{\Delta x \rightarrow 0} \sum_{k=0}^{\infty} \sum_{m}\left\|a_{x t}\right\|_{L^{\infty}\left(D_{k m}\right)} 2(\Delta x)(\Delta t)=\left\|a_{x t}\right\|_{L^{1}(E)} .
\end{aligned}
$$

It follows by (4.39) -(4.42) that

$$
L\left(J_{n+1}\right) \leq F\left(J_{n+1}\right) \leq M^{*}+O(1) M^{*} \omega(E)+O(\Delta x) .
$$

According to (4.38) and (4.43), we are able to choose T.V. $\left\{U_{0}, u_{1 B}, a_{B}\right\}$ and $\omega(E)$ sufficiently small such that $O(1) 2 K_{1} \cdot T . V .\left\{U_{0}, u_{1 B}, a_{B}\right\} \leq M^{*} / 3$ and $O(1) M^{*} \omega(E) \leq$ $M^{*} / 3$, and this implies that

$$
L\left(J_{n+1}\right) \leq F\left(J_{n+1}\right) \leq M^{*}
$$


as $\Delta x$ tends to zero. Therefore (4.39) holds for $k=n+1$. By induction on $n$, we show that $L\left(J_{n}\right)$ has a uniform bound for all $n \in \mathbb{N}$. Since the functional $L$ is equivalent to the total variation of $U_{\theta, \Delta x}^{\varepsilon}$, the total variation of $U_{\theta, \Delta x}^{\varepsilon}$ is uniformly bounded for all $t \geq 0$ and all sufficiently small $\Delta x>0$, so as well the $L^{\infty}$ norm of $U_{\theta, \Delta x}^{\varepsilon}$. It is worthy to indicate that $u_{\theta, \Delta x}^{\varepsilon}$ is well-defined for $t>0$ and $\Delta x \rightarrow 0$. We complete the proof. $\mathrm{L}$

It is remarked that the approximate solutions of (1.1) has the same result as that in Theorem 4.4. The following theorem is a consequence of Theorem 4.4 and the results in $[31]$.

THEOREM 4.5. Let $U_{\theta, \Delta x}^{\varepsilon}$ be the approximate solution to (1.2) for any $0<\varepsilon \ll 1$. Suppose that T.V. $\left\{U_{0}, u_{1 B}, a_{B}\right\}$ and $\omega(E)$ are sufficiently small. Then

(i) T.V. $\left\{U_{\theta, \Delta x}^{\varepsilon}(\cdot, t)\right\} \leq C_{1}\left(T . V .\left\{U_{0}, u_{1 B}, a_{B}\right\}+\omega\right)$, where $C_{1}$ is independent of $\theta$, $\Delta x$ and $\varepsilon$.

(ii) $T . V .\left\{U_{\theta, \Delta x}^{\varepsilon}(x, i \Delta t)\right\}+\sup _{x}\left[U_{\theta, \Delta x}^{\varepsilon}(x, i \Delta t)\right] \leq C_{2}\left(T . V .\left\{U_{0}, u_{1 B}, a_{B}\right\}+\omega\right)$, where $C_{2}$ is independent of $\theta, \Delta x, i \Delta t$ and $\varepsilon$.

(iii) $\int_{0}^{\infty}\left|U_{\theta, \Delta x}^{\varepsilon}\left(x, t^{\prime}\right)-U_{\theta, \Delta x}^{\varepsilon}(x, t)\right| d x \leq C_{3}\left(\left|t^{\prime}-t\right|+\Delta t\right)$, where $C_{3}$ is independent
of $\theta, \Delta x$ and $\varepsilon$. Similarly, the above inequalities also hold for the initial value problem (1.1).

Finally, we give the compactness of subsequences of $\left\{U_{\theta, \Delta x}^{\varepsilon}\right\}$ and $\left\{f\left(U_{\theta, \Delta x}^{\varepsilon}\right)\right\}$. Weak convergences of the residual and of the source will be proved in Section 5 .

THEOREM 4.6. [15] Let $\left\{U_{\theta, \Delta x}^{\varepsilon}\right\}$ be the approximate solution to (1.2) generated by the GGS. Then there exists a subsequence $\left\{U_{\theta, \Delta x_{i}}^{\varepsilon}\right\}$ of $\left\{U_{\theta, \Delta x}^{\varepsilon}\right\}$ such that $U_{\theta, \Delta x_{i}}^{\varepsilon}$ converges to some measurable function $U^{\varepsilon}(x, t)$ in $L_{l o c}^{1}(E)$. The result also holds for sequence $\left\{U_{\theta, \Delta x}\right\}$ obtained by letting $\varepsilon \rightarrow 0$, that is, there exists a subsequence of $\left\{U_{\theta, \Delta x}\right\}$ tending to some measurable function $U(x, t)$ in $L_{l o c}^{1}(E)$. Furthermore,

(i) $U^{\varepsilon}(x, t) \rightarrow U(x, t)$ in $L_{l o c}^{1}(E)$ as $\varepsilon \rightarrow 0$,

(ii) for every continuous function $f$, we have

$$
f\left(U_{\theta, \Delta x_{i}}^{\varepsilon}\right) \rightarrow f\left(U^{\varepsilon}(x, t)\right) \text { in } L_{l o c}^{1}(E) \text { as } \Delta x \rightarrow 0,
$$

and

$$
f\left(U^{\varepsilon}(x, t)\right) \rightarrow f(U(x, t)) \text { in } L_{l o c}^{1}(E) \text { as } \varepsilon \rightarrow 0 .
$$

Similarly, properties (i), (ii) also hold for the initial value problem (1.1).

By the results in Section 3, we notice that

$$
\left|u_{1 \theta, \Delta x}^{\varepsilon}(0, t)-u_{1 B}(t)\right| \leq\left|u_{1 B}^{i}-u_{1 B}(t)\right| \leq O(\Delta x)
$$

for any $t>0$ and $i \in \mathbb{N} \cup\{0\}$. This implies that $u_{1 \theta, \Delta x}^{\varepsilon}$ will match the boundary data $u_{1 B}(t)$ as $\Delta x$ tends to zero.

5. Weak convergence and global existence theorem. In this section we prove the main theorem of this paper. Again, since the analysis for problems (1.1) and (1.2) are similar, we only concentrate on the problem (1.2). According to the results in Section 4, it remains to establish the consistency of the scheme, the weak convergence of the source term and the entropy inequalities.

Before we begin with the consistency of the scheme, we recall Definition 1.1 that $u(x, t)$ is a weak solution to (1.2) if and only if $R_{\phi}(a, u)=0$ for all $\phi \in C_{c}^{1}(E)$. 
Hereinafter we let $u^{\varepsilon}:=u_{\theta, \Delta x}^{\varepsilon}$ be the approximate solution satisfying $u_{\theta, \Delta x}^{\varepsilon} \rightarrow u$ in $L_{l o c}^{1}(E)$, and let $a^{\varepsilon}$ denote $a_{\Delta x}^{\varepsilon}$. Since $a_{x}^{\varepsilon} g\left(a^{\varepsilon}, a_{x}^{\varepsilon}, a_{t}^{\varepsilon}, u^{\varepsilon}\right)$ may fail to converge to $a_{x} g\left(a, a_{x}, a_{t}, u\right)$ weakly, we use the approximation $b^{\varepsilon}:=b_{\Delta x}^{\varepsilon}$ instead of $a_{t}^{\varepsilon}$ and re-define the residual as

$$
\begin{aligned}
\widehat{R}_{\phi}\left(a^{\varepsilon}, b^{\varepsilon}, u^{\varepsilon}, E\right):= & \iint_{E} u^{\varepsilon} \phi_{t}+f\left(a^{\varepsilon}, u^{\varepsilon}\right) \phi_{x}+a_{x}^{\varepsilon} g\left(a^{\varepsilon}, \bar{a}^{\prime}, b^{\varepsilon}, u^{\varepsilon}\right) \phi d x d t \\
& +\int_{0}^{\infty} u_{0}(x) \phi(x, 0) d x+\int_{0}^{\infty} f(a, u)(0, t) \phi(0, t) d t
\end{aligned}
$$

where $u(0, t)$ satisfies $u(0, t)=\left(u_{1 B}(t), u_{2}^{\varepsilon}(0, t)\right)$ and

$$
\bar{a}^{\prime}(x)=\sum_{i=0}^{\infty} a_{x}\left(x, t_{i}\right) \chi_{\left\{t_{i} \leqslant t<t_{i+1}\right\}}, \quad(\chi \text { the characteristic function }) .
$$

Then, to prove that the limit $u$ is a weak solution to (1.2), it is sufficient to show for any $\phi \in C_{c}^{1}(E)$,

$$
\begin{aligned}
& \widehat{R}_{\phi}\left(a^{\varepsilon}, b^{\varepsilon}, u^{\varepsilon}, E\right) \rightarrow 0, \quad \text { as } \varepsilon, \Delta x \rightarrow 0, \\
& \iint_{E}\left(a_{x}^{\varepsilon} g\left(a^{\varepsilon}, \bar{a}^{\prime}, b^{\varepsilon}, u^{\varepsilon}\right)-a_{x} g\left(a, a_{x}, a_{t}, u\right)\right) \phi d x d t \rightarrow 0 \quad \text { as } \varepsilon, \Delta x \rightarrow 0 .
\end{aligned}
$$

First, we show (5.2). Applying the divergence theorem to $\widehat{R}_{\phi}\left(a^{\varepsilon}, b^{\varepsilon}, u^{\varepsilon}, E\right)$, together with Theorems 2.6, 3.4 and 4.5, we obtain

$$
\begin{aligned}
\widehat{R}_{\phi}\left(a^{\varepsilon}, b^{\varepsilon}, u^{\varepsilon}, E\right)= & -\sum_{i \geq 1} J_{\varepsilon}^{i}-\int_{0}^{\infty}\left(u^{\varepsilon}(x, 0)-u_{0}(x)\right) \phi(x, 0) d x \\
& -\int_{0}^{\infty}\left(f\left(a^{\varepsilon}, u^{\varepsilon}\right)(0, t)-f(a, u)(0, t)\right) \phi(0, t) d t+O(\Delta x) \\
= & -J_{\varepsilon}+O(\Delta x)+O(\Delta x) \cdot\left(T . V \cdot\left\{U_{0}, u_{1 B}, a_{B}\right\}+\omega\right),
\end{aligned}
$$

where

$$
\begin{aligned}
{\left[u^{\varepsilon}\right](x, i \Delta t) } & :=u^{\varepsilon}\left(x, t_{i}^{+}\right)-u^{\varepsilon}\left(x, t_{i}^{-}\right), \\
J_{\varepsilon}^{i}=J_{\varepsilon}^{i}(\theta, \Delta x, \phi) & :=\int_{-\infty}^{\infty}\left[u^{\varepsilon}\right](x, i \Delta t) \phi(x, i \Delta t) d x, \\
J_{\varepsilon}=J_{\varepsilon}(\theta, \Delta x, \phi) & :=\sum_{i \geq 1} J_{\varepsilon}^{i} .
\end{aligned}
$$

It remains to estimate $J_{\varepsilon}$. To this aim, we appeal to a result in [15].

THEOREM 5.1. (See [15]) Let $\left\{U_{\theta, \Delta x}^{\varepsilon}\right\}$ be a family of approximate solutions constructed by the GGS in Section 4. Then for any $0<\varepsilon \ll 1$ we can find a null set $N_{\varepsilon} \subset \Phi$ and a subsequence $\left\{\Delta x_{i}\right\} \rightarrow 0$ such that for any $\theta \in \Phi / N_{\varepsilon}$ and $\phi \in C_{c}^{1}(E)$, we have

$$
J_{\varepsilon}\left(\theta, \Delta x_{i}, \phi\right)=O(1) \cdot \varepsilon^{\frac{1}{2}} \quad \text { as } \Delta x_{i} \rightarrow 0 .
$$

Therefore, by (5.4) and Theorem 5.1, we obtain (5.2). 
Next, we show the weak convergence of the source, i.e., show (5.3). Given $\delta>$ 0 , let $g_{\delta}\left(a, a_{x}, a_{t}, u\right)$ be the mollification of $g\left(a, a_{x}, a_{t}, u\right)$, that is, $g_{\delta}\left(a, a_{x}, a_{t}, u\right):=$ $g\left(a, a_{x}, a_{t}, u\right) * \psi_{\delta}$ where $\psi_{\delta}$ is the standard mollifier and "*" denotes the convolution. Then, by the triangle inequality, we see that

$$
\left|\iint_{E} a_{x}^{\varepsilon} g\left(a^{\varepsilon}, \bar{a}^{\prime}, b^{\varepsilon}, u^{\varepsilon}\right)-a_{x} g\left(a, a_{x}, a_{t}, u\right) \phi d x d t\right| \leq I_{1}+I_{2}+I_{3}+I_{4},
$$

where

$$
\begin{aligned}
& I_{1}:=\left|\iint_{E} a_{x}^{\varepsilon}\left(g\left(a^{\varepsilon}, \bar{a}^{\prime}, b^{\varepsilon}, u^{\varepsilon}\right)-g_{\delta}\left(a^{\varepsilon}, \bar{a}^{\prime}, b^{\varepsilon}, u^{\varepsilon}\right)\right) \phi d x d t\right| \\
& I_{2}:=\left|\iint_{E}\left(a_{x}^{\varepsilon}-a_{x}\right) g_{\delta}\left(a^{\varepsilon}, \bar{a}^{\prime}, b^{\varepsilon}, u^{\varepsilon}\right) \phi d x d t\right| \\
& I_{3}:=\left|\iint_{E} a_{x}\left(g_{\delta}\left(a^{\varepsilon}, \bar{a}^{\prime}, b^{\varepsilon}, u^{\varepsilon}\right)-g\left(a^{\varepsilon}, \bar{a}^{\prime}, b^{\varepsilon}, u^{\varepsilon}\right)\right) \phi d x d t\right| \\
& I_{4}:=\left|\iint_{E} a_{x}\left(g\left(a^{\varepsilon}, \bar{a}^{\prime}, b^{\varepsilon}, u^{\varepsilon}\right)-g\left(a, a_{x}, a_{t}, u\right)\right) \phi d x d t\right| .
\end{aligned}
$$

Let

$E_{i 1}:=\left[0, x_{1}\right] \times\left[t_{i}, t_{i+1}\right], \quad E_{i k}:=\left[x_{k-1}, x_{k+1}\right] \times\left[t_{i}, t_{i+1}\right], \quad i=0,1,2, \ldots, k=2,4, \ldots$

To estimate $I_{1}$, we use the smoothness of standing waves, $\left|a_{x}^{\varepsilon}\right|=\frac{O(1)}{\varepsilon}\left|a_{x}\right|=\frac{O(1)}{\varepsilon}$ and $g_{\delta} \rightarrow g$ as $\delta \rightarrow 0$ at every point of continuity of $g$. Hence

$$
\begin{aligned}
I_{1} & \leq\left\|a_{x}^{\varepsilon}\right\|_{L^{\infty}}\|\phi\|_{L^{\infty}} \int_{0}^{T} \int_{0}^{L}\left|g\left(a^{\varepsilon}, \bar{a}_{x}, b^{\varepsilon}, u^{\varepsilon}\right)-g_{\delta}\left(a^{\varepsilon}, \bar{a}_{x}, b^{\varepsilon}, u^{\varepsilon}\right)\right| d x d t \\
& \leq \frac{C}{\varepsilon}\|\phi\|_{L^{\infty}}\left\|g-g_{\delta}\right\|_{L^{1}},
\end{aligned}
$$

where $[0, T] \times[0, L]$ is support of $\phi$ and, by the property of the mollification, $I_{1} \rightarrow 0$ as $\delta \rightarrow 0$. To estimate $I_{2}$, we use Taylor expansion of $g_{\delta}$ and $\partial^{\alpha} g_{\delta}=\partial^{\alpha}\left(g * \psi_{\delta}\right)=g * \partial^{\alpha} \psi_{\delta}$ for $\alpha=\left(\alpha_{1}, \alpha_{2}\right)$ so that $I_{2}$ can be written as

$$
\begin{aligned}
I_{2}= & \mid \sum_{i, k} \iint_{E_{i k}}\left(a_{x}^{\varepsilon}-a_{x}\right)\left[g_{\delta}\left(x_{k}, t_{i}\right)+\left(g * \partial_{x} \psi_{\delta}\right)\left(x_{k}, t_{i}\right)\left(x-x_{k}\right)\right. \\
& \left.+\left(g * \partial_{t} \psi_{\delta}\right)\left(x_{k}, t_{i}\right)\left(t-t_{i}\right)+\sum_{|\alpha|=2} \frac{\left(x-x_{k}, t-t_{i}\right)^{\alpha}}{\alpha !} \partial^{\alpha} g_{\delta}\left(\bar{x}_{k}, \bar{t}_{i}\right)\right] \phi d x d t \mid \\
\leq & \sum_{i, k}\left|g_{\delta}\left(x_{k}, t_{i}\right)\right| \cdot L_{i k}^{1}+\sum_{i, k} L_{i k}^{2}+O(\Delta x)
\end{aligned}
$$

where $\left(\bar{x}_{0}, \bar{t}_{i}\right) \in\left[0, x_{1}\right] \times\left[t_{i}, t_{i+1}\right],\left(\bar{x}_{k}, \bar{t}_{i}\right) \in\left[x_{k-1}, x_{k+1}\right] \times\left[t_{i}, t_{i+1}\right]$, and

$$
\begin{aligned}
L_{i k}^{1} & :=\left|\iint_{E_{i k}}\left(a_{x}^{\varepsilon}-a_{x}\right) \phi d x d t\right|, \\
L_{i k}^{2} & :=\left|\iint_{E_{i k}}\left(a_{x}^{\varepsilon}-a_{x}\right)\left[\left(g * \partial_{x} \psi_{\delta}\right)\left(x_{k}, t_{i}\right)\left(x-x_{k}\right)+\left(g * \partial_{t} \psi_{\delta}\right)\left(x_{k}, t_{i}\right)\left(t-t_{i}\right)\right] \phi d x d t\right| .
\end{aligned}
$$


Here we emphasize that it is necessary to use the mollification $g_{\delta}$ so that the Taylor expansion of $g_{\delta}$ can be apply to $I_{2}$. Now, $L_{i k}^{1}$ and $L_{i k}^{2}$ are estimated as follows. By the construction of $a^{\varepsilon}$ along with Taylor expansion of $\phi$, we have

$$
\begin{aligned}
L_{i k}^{1}= & \left|\phi\left(x_{k}, t_{i}\right)\right|\left|\int_{t_{i}}^{t_{i+1}}\left[a\left(x_{k+1}, t_{i}\right)-a\left(x_{k+1}, t\right)-a\left(x_{k-1}, t_{i}\right)+a\left(x_{k-1}, t\right)\right] d t\right| \\
& +O(\Delta x)^{2} \cdot \sup _{t_{i} \leq t \leq t_{i+1}}\left\{T . V \cdot\left\{a(\cdot, t) \text { in }\left[x_{k-1}, x_{k+1}\right]\right\}\right\} \\
= & \left|\phi\left(x_{k}, t_{i}\right)\right|\left|\int_{t_{i}}^{t_{i+1}}\left\{a_{t}\left(x_{k+1}, t_{i}\right)\left(t-t_{i}\right)-a_{t}\left(x_{k-1}, t_{i}\right)\left(t-t_{i}\right)\right\} d t\right|+O(\Delta x)^{3} \\
& +O(\Delta x)^{2} \cdot \sup _{t_{i} \leq t \leq t_{i+1}}\left\{T . V \cdot\left\{a(\cdot, t) \text { in }\left[x_{k-1}, x_{k+1}\right]\right\}\right\} \\
= & \left|\int_{t_{i}}^{t_{i+1}} a_{t x}\left(x_{k}^{*}, t_{i}\right)(2 \Delta x)\left(t-t_{i}\right) d t\right|+O(\Delta x)^{3} \\
& +O(\Delta x)^{2} \cdot \sup _{t_{i} \leq t \leq t_{i+1}}\left\{T . V \cdot\left\{a(\cdot, t) \text { in }\left[x_{k-1}, x_{k+1}\right]\right\}\right\} \\
= & O(\Delta x)^{3}+O(\Delta x)^{2} \sup _{t_{i} \leq t \leq t_{i+1}}\left\{T . V \cdot\left\{a(\cdot, t) \text { in }\left[x_{k-1}, x_{k+1}\right]\right\}\right\}
\end{aligned}
$$

where $x_{k}^{*} \in\left(x_{k-1}, x_{k+1}\right)$ and $k=2,4,6, \ldots$. Similarly, $L_{i 1}^{1}$ is estimated by

$$
L_{i 1}^{1}=O(\Delta x)^{3}+O(\Delta x)^{2} \cdot \sup _{t_{i} \leq t \leq t_{i+1}}\left\{T . V .\left\{a(\cdot, t) \text { in }\left[0, x_{1}\right]\right\}\right\} .
$$

By the properties of the mollifier, we have $\left(g * \partial^{\alpha} \psi_{\delta}\right)(x, t) \leq\|g\|_{L^{\infty}}\left\|\partial^{\alpha} \psi_{\delta}\right\|_{L^{1}}$ for $(x, t) \in E$, and it leads to

$$
\begin{aligned}
L_{i k}^{2} & =O(\Delta x)\|\phi\|_{\infty}\|g\|_{L^{\infty}}\left\|\partial \psi_{\delta}\right\|_{L^{1}} \iint_{E_{i k}}\left|a_{x}^{\varepsilon}-a_{x}\right| d x d t \\
& =O(\Delta x)^{2} \cdot \sup _{t_{i} \leq t \leq t_{i+1}}\left\{T . V \cdot\left\{a(\cdot, t) \text { in }\left[x_{k-1}, x_{k+1}\right]\right\}\right\} .
\end{aligned}
$$

Therefore, by (5.7) - (5.10), there exist positive constants $C_{1}$ and $C_{2}$ such that

$$
I_{2} \leq C_{1}(\Delta x)+C_{2}(\Delta x) \cdot \sup _{t \geq 0}\{T . V \cdot\{a(\cdot, t)\}\} .
$$

To estimate $I_{3}$, by the smoothness of $a$ and the property of mollifiers, we obtain

$$
I_{3} \leq C_{3}\|\phi\|_{L^{\infty}}\left\|g-g_{\delta}\right\|_{L^{1}} .
$$

Finally, we estimate $I_{4}$. We notice that $\left|\bar{a}^{\prime}-a_{x}\right|=O(\Delta x)$ and $\left|b^{\varepsilon}-a_{t}\right|=O(\Delta x)$ in each grid. Therefore, by the boundedness of $g_{a}, g_{a_{x}}, g_{a_{t}}, g_{u}$ and Theorem 4.6, we obtain

$$
\begin{aligned}
& I_{4} \leq O(1)\left(\left\|a^{\varepsilon}-a\right\|_{L_{l o c}^{1}}+\left\|u^{\varepsilon}-u\right\|_{L_{l o c}^{1}}\right)+O(\Delta x)\left(\left\|g_{a_{x}}\right\|_{\infty}+\left\|g_{a_{t}}\right\|_{\infty}\right) \iint_{E}\left|a_{x}\right| d x d t \\
& \quad \leq O(1)\left(\left\|a^{\varepsilon}-a\right\|_{L_{l o c}^{1}}+\left\|u^{\varepsilon}-u\right\|_{L_{l o c}^{1}}\right)+O(\Delta x) \sup _{t \geq 0}\{T . V .\{a(\cdot, t)\}\} .
\end{aligned}
$$

Finally, for any $0<\varepsilon \ll 1$ we choose such $\delta$ so that $\frac{1}{\varepsilon}\left\|g-g_{\delta}\right\|_{L^{1}} \rightarrow 0$ as $\varepsilon \rightarrow 0$. Then, by taking $\varepsilon$ and $\Delta x \rightarrow 0$ in (5.5)-(5.13), we obtain (5.3). We establish the global existence of weak solutions to the IBVP (1.2). 
In the end, we show that the weak solution $u$ is an entropy solution satisfying (1.16) for every entropy pair $(U(u), F(a, u))$. It is equivalent to show that, for any entropy pair $(U, F)$ and positive $\phi \in C_{c}^{1}(E)$, the approximate solution $u^{\varepsilon}$ satisfies the inequality

$$
\begin{aligned}
& \sum_{i, k} \widetilde{R}_{\phi}\left(a^{\varepsilon}, b^{\varepsilon}, u^{\varepsilon} ; E_{i k}\right)+\int_{0}^{\infty} U\left(u_{0}(x)\right) \phi(x, 0) d x+\int_{0}^{\infty} F(a, u)(0, t) \phi(0, t) d t \\
& \quad \geq O(\Delta x)+O(1) \varepsilon^{\frac{1}{2}}
\end{aligned}
$$

where

$\widetilde{R}_{\phi}\left(a^{\varepsilon}, b^{\varepsilon}, u^{\varepsilon} ; E_{i k}\right):=\iint_{E_{i k}}\left[U\left(u^{\varepsilon}\right) \phi_{t}+F\left(a^{\varepsilon}, u^{\varepsilon}\right) \phi_{x}+a_{x}^{\varepsilon}\left(D_{u} U\left(g-f_{a}\right)+F_{a}\right) \phi\right] d x d t$.

To prove (5.14), we need to calculate $\widetilde{R}_{\phi}$ in each grid $E_{i k}$. Write $E_{i k}=D_{i k}^{L} \cup D_{i k}^{\varepsilon} \cup D_{i k}^{R}$, where $D_{i k}^{L}:=\left\{(x, t): x_{k-1} \leq x<x_{k}-\varepsilon \widetilde{\Delta x}, t_{i} \leq t \leq t_{i+1}\right\}, D_{i k}^{\varepsilon}:=\{(x, t)$ : $\left.x_{k}-\varepsilon \widetilde{\Delta x} \leq x \leq x_{k}+\varepsilon \widetilde{\Delta x}, t_{i} \leq t \leq t_{i+1}\right\}$ and $D_{i k}^{R}:=\left\{(x, t): x_{k}+\varepsilon \widetilde{\Delta x}<x \leq\right.$ $\left.x_{k+1}, t_{i} \leq t \leq t_{i+1}\right\}$. Without loss of generality, we assume that there exist a single shock, denoted by $\mathcal{S}$, in $D_{i k}^{L}$ and a rarefaction wave in $D_{i k}^{R}$. In addition, the shock $\mathcal{S}$ divides $D_{i k}^{L}$ into $D_{i k}^{L^{-}}$and $D_{i k}^{L^{+}}$, and also connects the left state $u_{L}$ and the right state $u_{1}$. Also, let $a^{\varepsilon}=a_{L}, b^{\varepsilon}=b_{L}$ in $D_{i k}^{L}$ and $a^{\varepsilon}=a_{R}, b^{\varepsilon}=b_{R}$ in $D_{i k}^{R}$. Then, it is easy to see

$$
\begin{aligned}
\widetilde{R}_{\phi}\left(a^{\varepsilon}, b^{\varepsilon}, u^{\varepsilon} ; D_{i k}\right)= & \widetilde{R}_{\phi}\left(a_{L}, b_{L}, u^{\varepsilon} ; D_{i k}^{L}\right)+\widetilde{R}_{\phi}\left(a^{\varepsilon}, b^{\varepsilon}, u^{\varepsilon} ; D_{i k}^{\varepsilon}\right) \\
& +\widetilde{R}_{\phi}\left(a_{R}, b_{R}, u^{\varepsilon} ; D_{i k}^{R}\right) .
\end{aligned}
$$

First, we estimate $\widetilde{R}_{\phi}\left(a_{L}, b_{L}, u^{\varepsilon} ; D_{i k}^{L}\right)$. By $U\left(u^{\varepsilon}\right)=U\left(u_{L}\right)$ in $D_{i k}^{L^{-}}$and $U\left(u^{\varepsilon}\right)=$ $U\left(u_{1}\right)$ in $D_{i k}^{L^{+}}$, we have

$$
\begin{aligned}
\widetilde{R}_{\phi}\left(a_{L}, b_{L}, u^{\varepsilon} ; D_{i k}^{L}\right)= & \iint_{D_{i k}^{L^{-}}}\left(U\left(u_{L}\right) \phi\right)_{t}+\left(\left(F+(\Delta t) b_{L} F_{a}\right)\left(a_{L}, u_{L}\right) \phi\right)_{x} d x d t \\
& +\iint_{D_{i k}^{L^{+}}}\left(U\left(u_{1}\right) \phi\right)_{t}+\left(\left(F+(\Delta t) b_{L} F_{a}\right)\left(a_{L}, u_{1}\right) \phi\right)_{x} d x d t \\
& -\iint_{D_{i k}^{L^{-}} \cup D_{i k}^{L^{+}}}(\Delta t) b_{L} F_{a}\left(a_{L}, u^{\varepsilon}\right) \phi_{x} d x d t .
\end{aligned}
$$

Applying the divergence theorem to (5.16), we obtain

$$
\begin{aligned}
\widetilde{R}_{\phi}\left(a_{L}, b_{L}, u^{\varepsilon} ; D_{i k}^{L}\right)= & Y_{i k}(U, F)+\int_{\mathcal{S}} \phi\left(-U\left(u_{L}\right)+U\left(u_{1}\right)\right) d x \\
& +\int_{\mathcal{S}} \phi\left(\left(F+(\Delta t) b_{L} F_{a}\right)\left(a_{L}, u_{L}\right)-\left(F+(\Delta t) b_{L} F_{a}\right)\left(a_{L}, u_{1}\right)\right) d t \\
& -\iint_{D_{i k}^{L-} \cup D_{i k}^{L+}}(\Delta t) b_{L} F_{a}\left(a_{L}, u^{\varepsilon}\right) \phi_{x} d x d t,
\end{aligned}
$$


where

$$
\begin{aligned}
Y_{i k}(U, F):= & \int_{x_{k-1}}^{x_{k}-\varepsilon \widetilde{\Delta x}} U\left(u^{\varepsilon}\left(x, t_{i+1}\right)\right) \phi\left(x, t_{i+1}\right) d x-\int_{x_{k-1}}^{x_{k}-\varepsilon \widetilde{\Delta x}} U\left(u^{\varepsilon}\left(x, t_{i}\right)\right) \phi\left(x, t_{i}\right) d x \\
& +\int_{t_{i}}^{t_{i+1}}\left(F+(\Delta t) b_{L} F_{a}\right)\left(a_{L}, u^{\varepsilon}\left(x_{k}-\varepsilon \widetilde{\Delta x}, t\right)\right) \phi\left(x_{k}-\varepsilon \widetilde{\Delta x}, t\right) d t \\
& -\int_{t_{i}}^{t_{i+1}}\left(F+(\Delta t) b_{L} F_{a}\right)\left(a_{L}, u^{\varepsilon}\left(x_{k-1}, t\right)\right) \phi\left(x_{k-1}, t\right) d t .
\end{aligned}
$$

The Rankine-Hugoniot condition (2.16) gives

$$
s^{\Delta t}\left(u_{L}-u_{1}\right)=f^{\Delta t}\left(a_{L}, b_{L}, u_{L}\right)-f^{\Delta t}\left(a_{L}, b_{L}, u_{1}\right),
$$

where $f^{\Delta t}$ is given in (2.10) with $\delta=\Delta t$ and $s^{\Delta t}$ is the speed of $\mathcal{S}$. Furthermore, by the definition of $(U, F)$, we have $D_{u} F_{a}=\left(D_{u} U\right)\left(D_{u} f_{a}\right)$ and therefore

$$
D_{u}\left(F+(\Delta t) b_{L} F_{a}\right)=\left(D_{u} U\right)\left(D_{u} f^{\Delta t}\right) .
$$

Then, by (5.18), (5.19) and the results in [31], we obtain

$$
s^{\Delta t}\left[U\left(u_{L}\right)-U\left(u_{1}\right)\right]-\left[\left(F+(\Delta t) b_{L} F_{a}\right)\left(a_{L}, u_{L}\right)-\left(F+(\Delta t) b_{L} F_{a}\right)\left(a_{L}, u_{1}\right)\right] \leq 0 .
$$

By (5.17) and (5.20), we obtain

$$
\widetilde{R}_{\phi}\left(a_{L}, b_{L}, u^{\varepsilon} ; D_{i k}^{L}\right) \geq Y_{i k}(U, F)+O(\Delta x)^{3}
$$

for any positive test function $\phi \in C_{c}^{1}(E)$.

To compute $\widetilde{R}_{\phi}\left(a_{R}, b_{R}, u^{\varepsilon} ; D_{i k}^{R}\right)$, we notice that the rarefaction wave in $D_{i k}^{R}$ is a classical solution. It follows

$$
\begin{aligned}
& \widetilde{R}_{\phi}\left(a_{R}, b_{R}, u^{\varepsilon} ; D_{i k}^{R}\right) \\
& =\int_{x_{k}+\varepsilon \widetilde{\Delta x}}^{x_{k+1}} U\left(u^{\varepsilon}\left(x, t_{i+1}\right)\right) \phi\left(x, t_{i+1}\right) d x-\int_{x_{k}+\varepsilon \widetilde{\Delta x}}^{x_{k+1}} U\left(u^{\varepsilon}\left(x, t_{i}\right)\right) \phi\left(x, t_{i}\right) d x \\
& \quad+\int_{t_{i}}^{t_{i+1}}\left(F+(\Delta t) b_{R} F_{a}\right)\left(a_{R}, u^{\varepsilon}\left(x_{k+1}, t\right)\right) \phi\left(x_{k+1}, t\right) d t \\
& \quad-\int_{t_{i}}^{t_{i+1}}\left(F+(\Delta t) b_{R} F_{a}\right)\left(a_{R}, u^{\varepsilon}\left(x_{k}+\varepsilon \widetilde{\Delta x}, t\right)\right) \phi\left(x_{k}+\varepsilon \widetilde{\Delta x}, t\right) d t
\end{aligned}
$$

for any positive test function $\phi \in C_{c}^{1}(E)$.

Finally, we calculate $\widetilde{R}_{\phi}\left(a^{\varepsilon}, b^{\varepsilon}, u^{\varepsilon} ; D_{i k}^{\varepsilon}\right)$. Since $U\left(u^{\varepsilon}\right)$ is only a function of $x$ in $D_{i k}^{\varepsilon}$, by the integration by parts, the definition of $(U, F)$ and $D_{u} F_{a}=\left(D_{u} U\right)\left(D_{u} f_{a}\right)$, 
we obtain

$$
\begin{aligned}
\widetilde{R}_{\phi}\left(a^{\varepsilon}, b^{\varepsilon}, u^{\varepsilon} ; D_{i k}^{\varepsilon}\right) & \\
= & \iint_{D_{i k}^{\varepsilon}}\left[U\left(u^{\varepsilon}\right) \phi_{t}+F\left(a^{\varepsilon}, u^{\varepsilon}\right) \phi_{x}+(\Delta t) b\left(x_{k}\right) F_{a} \phi_{x}-(\Delta t) b\left(x_{k}\right) F_{a} \phi_{x}\right] d x d t \\
& +\iint_{D_{i k}^{\varepsilon}} a_{x}^{\varepsilon}\left(D_{u} U\left(u^{\varepsilon}\right)\left(g-f_{a}\right)+F_{a}\right) \phi d x d t \\
= & Z_{i k}(U, F)-\iint_{D_{i k}^{\varepsilon}}\left[\left(D_{u} F\right) u_{x}^{\varepsilon}-a_{x}^{\varepsilon}\left(D_{u} U\right)\left(g-f_{a}\right)\right] \phi d x d t \\
& -\iint_{D_{i k}^{\varepsilon}}\left(\Delta t \cdot b\left(x_{k}\right) F_{a}\right)_{x} \phi d x d t+O(\Delta x)^{3} \\
= & Z_{i k}(U, F)-\iint_{D_{i k}^{\varepsilon}} D_{u} U\left[\left(D_{u} f\right) u_{x}^{\varepsilon}-a_{x}^{\varepsilon}\left(g-f_{a}\right)\right] \phi d x d t \\
& -(\Delta t) b\left(x_{k}\right) \iint_{D_{i k}^{\varepsilon}}\left[F_{a a} a_{x}^{\varepsilon}+\left(D_{u} U\right)\left(D_{u} f_{a}\right) u_{x}^{\varepsilon}\right] \phi d x d t+O(\Delta x)^{3} \\
= & Z_{i k}(U, F)-\iint_{D_{i k}^{\varepsilon}} D_{u} U\left(u^{\varepsilon}\right)\left[\left(f+(\Delta t) b\left(x_{k}\right) f_{a}\right)_{x}-a_{x}^{\varepsilon} g\right] \phi d x d t \\
& +(\Delta t) b\left(x_{k}\right) \iint_{D_{i k}^{\varepsilon}} a_{x}^{\varepsilon}\left[D_{u} U\left(u^{\varepsilon}\right) f_{a a}-F_{a a}\right] \phi d x d t+O(\Delta x)^{3},
\end{aligned}
$$

where

$$
\begin{aligned}
Z_{i k}(U, F):= & \int_{x_{k}-\varepsilon \widetilde{\Delta x}}^{x_{k}+\varepsilon \widetilde{\Delta x}} U\left(u^{\varepsilon}\left(x, t_{i+1}\right)\right) \phi\left(x, t_{i+1}\right) d x-\int_{x_{k}-\varepsilon \widetilde{\Delta x}}^{x_{k}+\varepsilon \widetilde{\Delta x}} U\left(u^{\varepsilon}\left(x, t_{i}\right)\right) \phi\left(x, t_{i}\right) d x \\
& +\int_{t_{i}}^{t_{i+1}}\left(F+(\Delta t) b\left(x_{k}\right) F_{a}\right)\left(a_{R}, u^{\varepsilon}\left(x_{k}+\varepsilon \widetilde{\Delta x}, t\right)\right) \phi\left(x_{k}+\varepsilon \widetilde{\Delta x}, t\right) d t \\
& -\int_{t_{i}}^{t_{i+1}}\left(F+(\Delta t) b\left(x_{k}\right) F_{a}\right)\left(a_{L}, u^{\varepsilon}\left(x_{k}-\varepsilon \widetilde{\Delta x}, t\right)\right) \phi\left(x_{k}-\varepsilon \widetilde{\Delta x}, t\right) d t .
\end{aligned}
$$

It is easy to see

$$
\iint_{D_{i k}^{\varepsilon}} a_{x}^{\varepsilon}\left[D_{u} U\left(u^{\varepsilon}\right) f_{a a}-F_{a a}\right] \phi d x d t=O(\Delta x) \text { osc. }\left\{a^{\varepsilon}\left(\cdot, t_{i}\right) \text { in } D_{i k}^{\varepsilon}\right\} .
$$

In addition, by (2.19), we have

$$
\iint_{D_{i k}^{\varepsilon}} D_{u} U\left(u^{\varepsilon}\right)\left[\left(f+(\Delta t) b\left(x_{k}\right) f_{a}\right)_{x}-a_{x}^{\varepsilon} g\right] \phi d x d t=O(\Delta x)^{3} .
$$

Then, by (5.15) and (5.21)-(5.25), we obtain

$$
\begin{aligned}
\widetilde{R}_{\phi}\left(a^{\varepsilon}, b^{\varepsilon}, u^{\varepsilon} ; E_{i k}\right) \geq & \int_{x_{k-1}}^{x_{k+1}} U\left(u^{\varepsilon}\left(x, t_{i+1}\right)\right) \phi\left(x, t_{i+1}\right) d x-\int_{x_{k-1}}^{x_{k+1}} U\left(u^{\varepsilon}\left(x, t_{i}\right)\right) \phi\left(x, t_{i}\right) d x \\
& +\int_{t_{i}}^{t_{i+1}}\left(F+(\Delta t) b_{R} F_{a}\right)\left(a_{R}, u^{\varepsilon}\left(x_{k+1}, t\right)\right) \phi\left(x_{k+1}, t\right) d t \\
& -\int_{t_{i}}^{t_{i+1}}\left(F+(\Delta t) b_{L} F_{a}\right)\left(a_{L}, u^{\varepsilon}\left(x_{k-1}, t\right)\right) \phi\left(x_{k-1}, t\right) d t+O(\Delta x)^{3} \\
& +O(\Delta x)^{2}\left(\text { osc. }\left\{a^{\varepsilon}\left(\cdot, t_{i}\right) \text { in } E_{i k}\right\}+\text { osc. }\left\{a_{t}\left(\cdot, t_{i}\right) \text { in } E_{i k}\right\}\right)
\end{aligned}
$$


for any positive test function $\phi \in C_{c}^{1}(E)$.

It follows by (5.26) and the similar results in [15] that

$$
\begin{aligned}
\sum_{i, k} & \widetilde{R}_{\phi}\left(a^{\varepsilon}, b^{\varepsilon}, u^{\varepsilon} ; E_{i k}\right)+\int_{0}^{\infty} U\left(u_{0}(x)\right) \phi(x, 0) d x+\int_{0}^{\infty} F(a, u)(0, t) \phi(0, t) d t, \\
\geq & -\widehat{J}_{\varepsilon}-\int_{0}^{\infty}\left(U\left(u^{\varepsilon}(x, 0)\right)-U\left(u_{0}(x)\right)\right) \phi(x, 0) d x \\
& -\int_{0}^{\infty}\left(F\left(a^{\varepsilon}, u^{\varepsilon}\right)(0, t)-F(a, u)(0, t)\right) \phi(0, t) d t+O(\varepsilon+\Delta x) \\
& \geq-\widehat{J}_{\varepsilon}+O(\varepsilon+\Delta x),
\end{aligned}
$$

where

$$
\begin{gathered}
\widehat{J}_{\varepsilon}=\widehat{J}_{\varepsilon}(\theta, \Delta x, \phi):=\sum_{i \geq 1} \int_{-\infty}^{\infty}\left[U\left(u^{\varepsilon}\right)\right](x, i \Delta t) \phi(x, i \Delta t) d x, \\
{\left[U\left(u^{\varepsilon}\right)\right](x, i \Delta t):=U\left(u^{\varepsilon}\right)\left(x, t_{i}^{+}\right)-U\left(u^{\varepsilon}\right)\left(x, t_{i}^{-}\right) .}
\end{gathered}
$$

Using (5.27) and replacing $J_{\varepsilon}$ by $\widehat{J}_{\varepsilon}$ in Theorem 5.1, we obtain (5.14). Moreover, the weak convergence of $\left\{a_{x}^{\varepsilon}\left(D_{u} U\left[g-f_{a}\right]+F_{a}\right)\left(a^{\varepsilon}, \bar{a}^{\prime}, b^{\varepsilon}, u^{\varepsilon}\right)\right\}$ can be obtained by using the similar argument for $\left\{a_{x}^{\varepsilon} g\left(a^{\varepsilon}, \bar{a}^{\prime}, b^{\varepsilon}, u^{\varepsilon}\right)\right\}$. We then establish the existence of global entropy solutions to (1.2). The global existence result for (1.1) can also be obtained in the similar way. We accomplish the main theorem of this paper.

\section{REFERENCES}

[1] D. AmAdoRI, Initial-boundary value problems for nonlinear systems of conservation laws, NoDEA Nonlinear Diff. Equ. Appl., 4 (1997), pp. 1-42.

[2] D. Amadori and R. M. Colombo, Continuous dependence for $2 \times 2$ conservation laws with boundary, J. Diff. Equ., 138 (1997), pp. 229-266.

[3] D. Amadori, L. Gosse and G. Guerra, Global BV entropy solutions and uniqueness for hyperbolic systems of balance laws, Arch. Rational Mech. Anal., 162 (2002), pp. 327-366.

[4] Y. Chang, J. M. Hong And C.-H. Hsu, Globally Lipschitz continuous solutions to a class of quasilinear wave equations, J. Diff. Equ., 236 (2007), pp. 504-531.

[5] C. M. Dafermos And L. HsiaO, Hyperbolic systems of balance laws with inhomogeneity and dissipation, Indiana U. Math. J., 31 (1982), pp. 471-491.

[6] G. Dal Maso, P. LeFloch and F. Murat, Definition and Weak Stability of Nonconservative Products, J. Math. Pure Appl., 74 (1995), pp. 483-548.

[7] F. Dubois and P. G. LeFloch, Boundary conditions for nonlinear hyperbolic systems of conservation laws, J. Diff. Equ., 71 (1988), pp. 93-122.

[8] H. FRID, Initial-boundary value problems for conservation laws, J. Diff. Eqn., 128 (1996), pp. $1-45$.

[9] J. Gurmm, Solutions in the large for nonlinear hyperbolic systems of equations, Comm. Pure Appl. Math., 18 (1965), pp. 697-715.

[10] J. B. Goodman, Initial boundary value problem for hyperbolic systems of conservation laws, Ph.D. dissertation, Stanford University, 1982.

[11] J. Groah, J. Smoller and B. Temple, Shock Wave Interactions in General Relativity, Monographs in Mathematics, Springer, Berlin, New York, 2007.

[12] J. M. Hong, An extension of Glimm's method to inhomogeneous strictly hyperbolic systems of conservation laws by "weaker than weak" solutions of the Riemann problem, J. Diff. Equ., 222 (2006), pp. 515-549.

[13] J. M. Hong and P. G. LeFloch, A version of Glimm method based on generalized Riemann problems, J. Portugal Math., 64 (2007), pp. 199-236.

[14] J. M. Hong, C.-H. Hsu And Y.-C. Su, Global solutions for initial-boundary value problem of quasilinear wave equations, J. Diff. Equ., 245 (2008), pp. 223-248. 
[15] J. M. Hong AND Y.-C. Su, Generalized Glimm scheme to the initial boundary value problem of hyperbolic systems of balance laws, Nonlinear Anal-Theor, 72 (2010), pp. 635-650.

[16] J. M. Hong And B. Temple, A bound on the total variation of the conserved quantities for solutions of a general resonant nonlinear balance law, SIAM J. Appl. Math., 64:3 (2004), pp. 819-857.

[17] E. Isaacson and B. Temple, Nonlinear resonance in systems of conservation laws, SIAM J. Appl. Anal., 52 (1992), pp. 1260-1278.

[18] E. ISAACSON AND B. Temple, Convergence of the $2 \times 2$ Godunov method for a general resonant nonlinear balance law, SIAM J. Appl. Math., 55:3 (1995), pp. 625-640.

[19] W.-L. JIN, A kinematic wave theory of lanes-changing traffic flow, to appear in Transportation research, Part B.

[20] B. Keyfitz And H. Kranzer, A system of non-strictly hyperbolic conservation laws arising in elasticity theory, Arch. Ration. Mech. Anal., 72 (1980), pp. 219-241.

[21] P. D. Lax, Hyperbolic system of conservation laws, II, Comm. Pure Appl. Math., 10 (1957), pp. 537-566.

[22] P. G. LeFloch, Entropy Weak Solutions to Nonlinear Hyperbolic Systems Under Nonconservative Form, Comm. Part. Diff. Equ., 13 (1988), pp. 669-727.

[23] P. G. LeFloch, Shock Waves for Nonlinear Hyperbolic Systems in Nonconservative Form, Institute for Math. and its Appl., Minneapolis, Preprint 593 (1989).

[24] P. G. LeFloch And T.-P. Liu, Existence theory for nonlinear hyperbolic systems in nonconservative form, Forum Math., 5 (1993), pp. 261-280.

[25] T.-P. LiU, Initial-boundary value problems for gas dynamics, Arch. Rat. Mech. Anal., 64 (1977), pp. 137-168.

[26] T.-P. Liu, Quasilinear hyperbolic systems, Comm. Math. Phys., 68 (1979), pp. 141-172.

[27] T.-P. LiU, Approximation and qualitative behavior of admissible solutions of hyperbolic conservation laws, Mem. Amer. Math. Soc.

[28] T. Nishida And J. Smoller, Mixed problems for nonlinear conservation laws, J. Diff. Equ., 23 (1977), pp. 244-269.

[29] Y.-C. Su, Global entropy solutions to a class of quasi-linear wave equations with large timeoscillating sources, J. Diff. Equ., 250:9, (2011), pp. 3668-3700.

[30] B. Temple, Global solution of the Cauchy problem for a class of $2 \times 2$ nonstrictly hyperbolic conservation laws, Adv. Appl. Math., 3 (1982), pp. 335-375.

[31] J. Smoller, Shock Waves and Reaction-Diffusion Equations, 2nd ed., Springer-Verlag, Berlin, New York, 1994.

[32] B. Whiтнам, Linear and nonlinear waves, New York, John Wiley, 1974. 